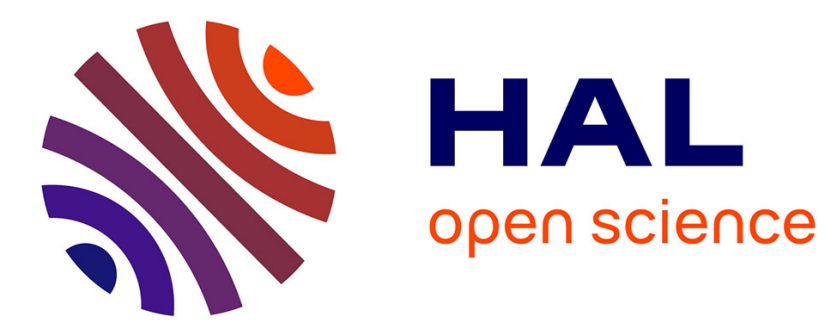

\title{
De la naissance à l'oubli d'un quartier antique de Vannes (Morbihan), les résultats d'une fouille de sauvetage au 10 rue de la Tannerie
}

Gaétan Le Cloirec

\section{- To cite this version:}

Gaétan Le Cloirec. De la naissance à l'oubli d'un quartier antique de Vannes (Morbihan), les résultats d'une fouille de sauvetage au 10 rue de la Tannerie. Revue Archéologique de l'Ouest, 1998, 15, pp.151-171. 10.3406/rao.1998.1076 . hal-02427629

\section{HAL Id: hal-02427629 \\ https://hal.science/hal-02427629}

Submitted on 17 Jan 2020

HAL is a multi-disciplinary open access archive for the deposit and dissemination of scientific research documents, whether they are published or not. The documents may come from teaching and research institutions in France or abroad, or from public or private research centers.
L'archive ouverte pluridisciplinaire HAL, est destinée au dépôt et à la diffusion de documents scientifiques de niveau recherche, publiés ou non, émanant des établissements d'enseignement et de recherche français ou étrangers, des laboratoires publics ou privés. 


\title{
De la naissance à l'oubli d'un quartier antique de Vannes (Morbihan) : Les résultats d'une fouille de sauvetage au 10, rue de la Tannerie
}

In: Revue archéologique de l'ouest, tome 15, 1998. pp. 151-171.

\begin{abstract}
Résumé
Les étapes du développement urbain de la ville antique de Vannes ont pu être abordées à l'occasion d'une fouille de sauvetage réalisée en 1993 au 10, rue de la Tannerie. L'occupation gallo-romaine s'y épanouit entre le règne d'Auguste et la fin du Ille siècle de notre ère. Aux premiers aménagements sporadiques succède une organisation orthogonale établie à partir d'un espace de circulation qui divise le site. L'architecture qui prend place dans ce cadre subit également une évolution dont l'apogée pourrait correspondre à l'établissement d'une imposante construction à galeries. L'occupation médiévale est marquée par une activité artisanale éventuellement liée à la tannerie alors que des zones d'extraction perturbent ponctuellement le terrain. Le site est ensuite occupé par un jardin associé à des constructions établies dans la partie nord-ouest.
\end{abstract}

\section{Abstract}

The urban development of roman Vannes has been recently studied by excavation at $n^{\circ} 10$, rue de la Tannerie. Roman occupation has been attested, being situated between the period of August and the end of the third century A.D. The earliest phase of nonorganised fight structures is followed by the laying out of a regular street plan. The architecture established in this setting evolves likewise, terminating with the installation of a large galleried building. Medieval occupation is represented by an artisanal activity which could be linked with leather working. During the same period quarrying material occurs on the site. The site is occupied during the post- medieval and modem periods by a garden and buildings.

Citer ce document / Cite this document :

Le Cloirec Gaétan. De la naissance à l'oubli d'un quartier antique de Vannes (Morbihan) : Les résultats d'une fouille de sauvetage au 10, rue de la Tannerie. In: Revue archéologique de l'ouest, tome 15, 1998. pp. 151-171.

doi : 10.3406/rao.1998.1076

http://www.persee.fr/web/revues/home/prescript/article/rao_0767-709X_1998_num_15_1_1076 


\title{
DE LA NAISSANCE À L'OUBLI D'UN QUARTIER ANTIQUE DE VANNES (Morbihan) : Les résultats d'une fouille de sauvetage au 10, rue de la Tannerie
}

Gaétan LE CLOIREC*

\begin{abstract}
Résumé : Les étapes du développement urbain de la ville antique de Vannes ont pu être abordées à l'occasion d'une fouille de sauvetage réalisée en 1993 au 10, rue de la Tannerie. L'occupation gallo-romaine s'y épanouit entre le règne d'Auguste et la fin du IIIe siècle de notre ère. Aux premiers aménagements sporadiques succède une organisation orthogonale établie à partir d'un espace de circulation qui divise le site. L'architecture qui prend place dans ce cadre subit également une évolution dont l'apogée pourrait correspondre à l'établissement d'une imposante construction à galeries. L'occupation médiévale est marquée par une activité artisanale éventuellement liée à la tannerie alors que des zones d'extraction perturbent ponctuellement le terrain. Le site est ensuite occupé par un jardin associé à des constructions établies dans la partie nord-ouest.
\end{abstract}

\begin{abstract}
The urban development of roman Vannes has been recently studied by excavation at $n^{\circ} 10$, rue de la Tannerie. Roman occupation has been attested, being situated between the period of August and the end of the third century A.D. The earliest phase of nonorganised fight structures is followed by the laying out of a regular street plan. The architecture established in this setting evolves likewise, terminating with the installation of a large galleried building. Medieval occupation is represented by an artisanal activity which could be linked with leather working. During the same period quarrying material occurs on the site. The site is occupied during the postmedieval and modern periods by a garden and buildings.
\end{abstract}

Mots-clés : Urbanisme, îlot, terrasse, voirie, caniveau, bâtiment.

Key-words : Town planning, islet, terrace, refuse-dump, gutter, building.

Depuis quelques années, les quartiers de Vannes situés au nord-est de la ville close bénéficient d'une réhabilitation marquée par la construction d'ensembles nouveaux. A chaque fois, ces travaux donnent lieu à des interventions archéologiques d'ampleurs diverses puisque ce secteur correspond à l'emplacement de la ville antique du Haut Empire à laquelle ont succédé des quartiers médiévaux et modernes relativement importants. C'est dans $œ$ contexte particulièrement délicat que la construction d'un immeuble a été envisagée au 10, rue de la Tannerie. Les deux parcelles concernées par le projet représentent une surface de $1000 \mathrm{~m}^{2}$ dans une emprise sensible d'au moins 40 hectares (fig. 1). Une campagne de sondages ayant confirmé la présence de vestiges, une fouille de sauvetage s'est déroulée entre le 15 juillet et le 30 septembre 1993 afin de libérer le terrain des contraintes archéologiques. Les résultats de cette opération s'inscrivent dans une approche des origines et du développement de l'antique cité vénète. Mais la place du site dans le phénomène urbain ne peut être abordée sans prendre en compte le cadre naturel particulier de la ville de Vannes. De même, l'apparition et l'évolution de l'urbanisme doivent être mises en relation avec un environnement archéologique et historique clairement rappelé auparavant pour ce secteur précis de l'agglomération.

\section{CONTEXTE GÉOGRAPHIQUE ET HISTORIQUE : ÉTAT DES IDÉES SUR LA QUESTION (fig. 1)}

\section{CADRE GÉOGRAPHIQUE}

Situé en bout d'estuaire au fond du golfe du Morbihan, le paysage vannetais se caractérise par un relief composé de trois collines granitiques : Le Méné, Boismoreau et la Garenne. Dans l'emprise occupée par les villes antique, médiévale et moderne ces hauteurs culminent respectivement à $23 \mathrm{~m}, 17 \mathrm{~m}$ et $27 \mathrm{~m}$. Deux cours d'eau coulent à leur 


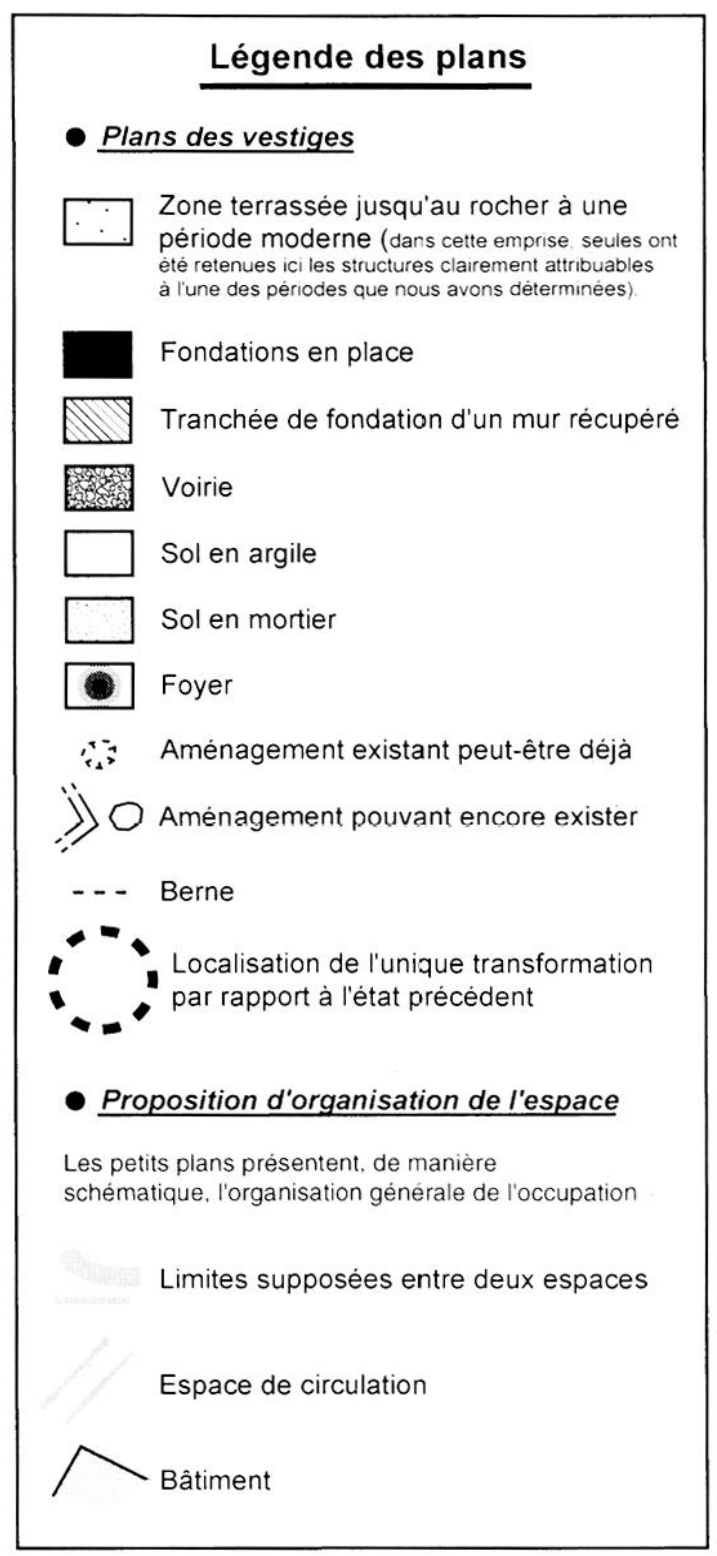

pied avant de se jeter dans la mer. De nombreux aménagements réalisés par l'Homme ainsi que d'importants apports alluvionnaires ont modelé peu à peu le paysage actuel. Il y a 2000 ans, en effet. la mer atteignait la base des collines et s'engoufrait profondément entre elles, alimentant ainsi des vasières importantes. Dans ce paysage ancien, la colline du Méné devait également présenter un aspect bcaucoup plus abrupt qu'aujourd'hui (Leguay, 1988, 5-7 et 1617).

Les parcelles du 10 , rue de la tannerie se placent sur le versant sud-cst de Boismoreau à mi-distance cntre le début du platcau et la base de la colline. Cette localisation confêre un pendage relativement marqué et régulier au terrain (environ $1 \mathrm{~m}$ pour $10 \mathrm{~m}$ ) tout en permettant un ensoleillement important une grande partic de la journce.

\section{CADRE HISTORIQUE}

Aucunc trace antćricurc à l'époque gallo-romaine n'a jamais été misc en évidence à Vannes. Seul le nom antique de la Cité, Darioritum, fait référence à la culture celtique puisqu'il dérive d'un toponyme gaulois (ritum) qui signific " gué ". Une création ex nihilo après la conquête romainc est donc supposćc.
Les vestiges les plus anciens remontent à la période augustéenne (fin Ier s. av. J.-C. / début Ier s. ap. J.-C.). Le sommet de la colline de Boismoreau est alors recoupé par plusieurs fossés éventuellement destinés à assainir le terrain avant l'aménagement d'une vaste esplanade recouverte de cailloutis. Dans la partie est, quelques constructions sur potcaux porteurs appartiendraient peut-être aux premiers habitats (catalogue Vannes, 1992, 92-93). Très rapidement, un quartier artisanal va se mettre en place à l'ouest de cet ensemble. Il a été reconnu sur les sites de la rue Sainte Catherine et de la ruelle du recteur (catalogue Vannes, 1992, 88-91). Au même moment une zone de stockage avec entrepôt (?) apparaîtrait sur le versant sud de la colline (site de la rue du four). La proximité de la mer et l'existence supposée d'un port expliqueraient ici la découverte de telles installations (catalogue Vannes, 1992, 8889).

Le développement de l'urbanisme se ferait à partir du $2 e$ quart du Ier siècle de notre ère sur la colline de Boismoreau où un ensemble monumental serait alors construit sur la hauteur (catalogue Vannes, 1992, 30 et 94). Dans la seconde partie du siècle, plusieurs habitations se développeraient autour de ce complexe conférant à la ville une extension maximale qu'on évalue actuellement à 40 hectares. Mais seule la mise en évidence d'une nécropole au sud-est permet d'estimer une limite urbaine de ce côté (fig. 1) (De Cusse, 1877).

La pćriode de troubles politiques et économiques qui marque la seconde moitié du IIIc siècle modifie profondément le paysage urbain. Certainement plus facile à défendre, la colline du Mené est peut-être fortifice dès cette époque, engendrant ainsi le déclin du site de Boismoreau (Catalogue Vannes, 1992, 69-70). L'établissement d'une garnison confirme cependant l'importance stratégique de la cité au Bas-Empire (Marsille, 1982).

La ville médiévale se développe à l'intérieur de la muraille alors que la ville du Haut-Empire est devenue un faubourg en ruines. A partir du VIe sic̀cle, une église y accueille les reliques de Saint Patern provoquant l'épanouissement d'un nouveau quartier sur une partie de l'ancienne ville gallo-romaine. Durant le Haut Moyen Age, la situation demeure relativement instable. La ville est même saccagée par les vikings à deux reprises, en 865 et en 919 (Leguay, 1988, 41 et 45). L'ćglise Saint Patern, très endommagée à cette dernière occasion, est reconstruite au XIe siècle. Mais un tremblement de terre survenu en 1286 occasionne à nouveau de tcrribles dégâts aux constructions (Leguay 1988, 41); les vestiges de la ville antique l'ont certainement encore moins bien supporté. Le nouvel essor urbain qui marque pourtant le XIIIe siècle est souligné par l'agrandissement des remparts et la construction de nouvcaux bâtiments. Pour cela de nombreuses carrières sont ouvertes dans les zones périurbaines. Malheureusement, la guerre de Succession de Bretagne (1341-1365) brise ce développement. La ville sort très meurtrie des quatre assauts qu'clle a subis durant ce conflit et les faubourgs, dont celui de Saint Patern. sont complètement détruits. Vannes connaît pourtant une nouvelle croissance sous la dỳnastic des Montfort entre le XIVe et le XVe siècle. De nouvelles constructions remettent la cité en état et lui apportent une image de ville prospère. Alors que d'importants remaniements modifient l'urbanisme de la ville close, des manoirs sont construits dans le faubourg de Saint Patern qui s'étend désormais sur une grande partie de la colline de Boismorcau. Néanmoins, le quartier demeure essenticllement rural (fermes, champs, pâtures) ct artisanal (tanneries, parcheminerics, clouterics, forges. moulins. ctc.). 


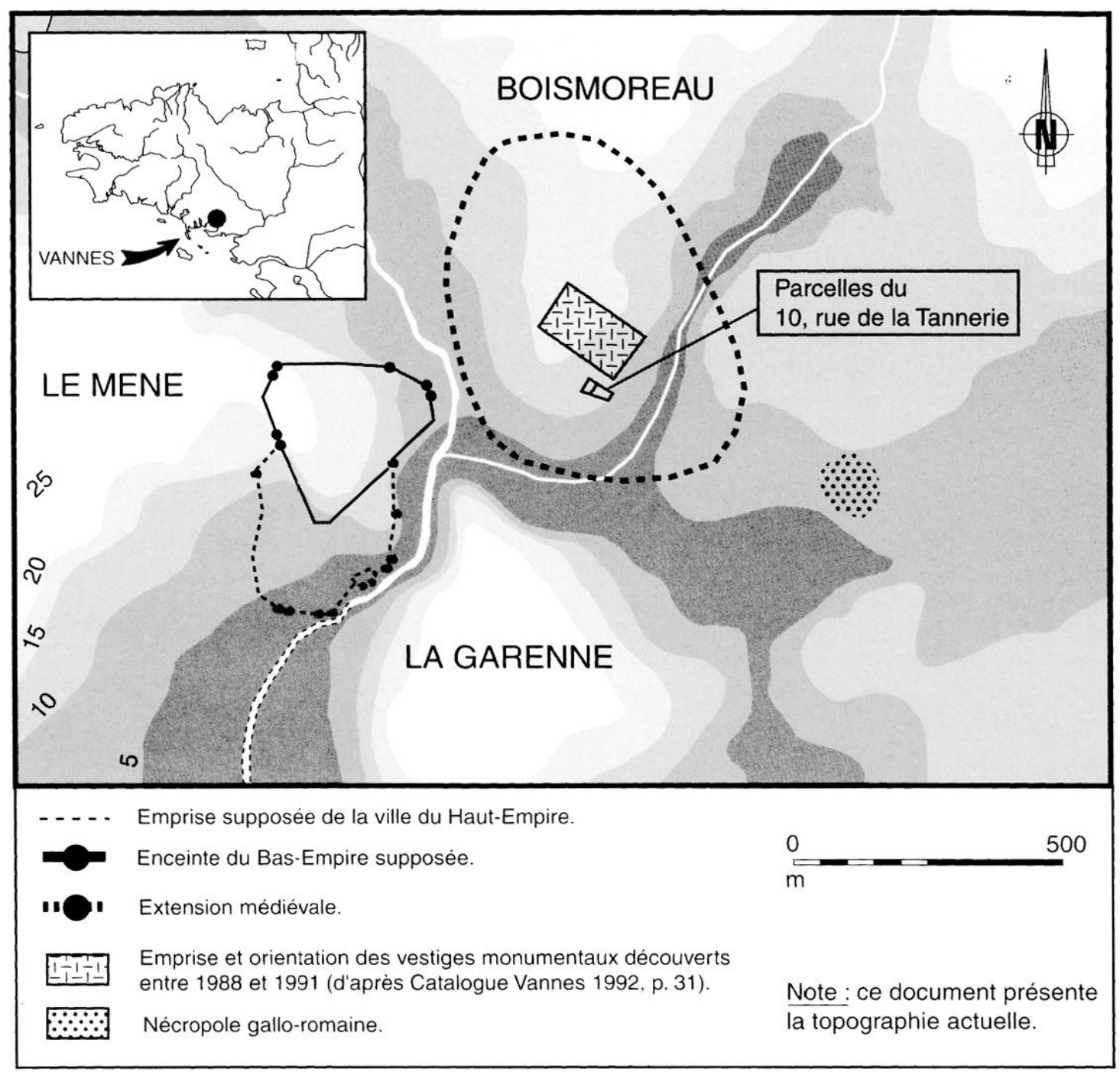

Fig. 1 : Contexte géographique de la ville antique de lannes.

Les troubles qui accompagnent le rattachement de la Bretagne à la France à la fin du XVe siècle provoquent, à nouveau, la destruction de Saint Patern. L'ćglise est remise en état mais, devenue trop petite au XVIIIe siòcle, clle est reconstruite une nouvelle fois. Ces derniers travaux correspondent au développement de l'urbanisme dans la partic de la ville situće au nord-cst de l'enceinte. La tanneric s'y ćpanouit au cours du XIXe sic̀cle alimentant une consommation essentiellement locale. Mais la seconde partic du sic̀cle correspond à une dégradation de l'image du quarticr qui devient plus populaire. De nombreux débits de boissons et des maisons closes y prolifèrent rapidement alors que les gens s'entassent dans des immeubles vétustes. L'établissement d'une garnison à proximité accompagne cette transformation et l'cxplique même certainement en partic.

Comme la ville close. le quartier Saint Patern bénéficic depuis les annécs 1980 d'une réhabilitation qui entraîne la restauration de bâtiments anciens ou la construction d'ensembles nouvcaux.

Les différentes étapes de l'urbanisme de la ville de Vannes sont done clairement liées à des périodes de développement, de menaces et de déclin. Dans les trois cas, l'organisation topographique du site prend une place essenticlle. L'agglomération initiale cst ainsi ćtablic sur un pla- tcau à proximitć d'un port d'ćchouage supposé. Plus tard, les dangers qui accompagnent l'affaiblissement puis la dislocation du cadre administratif romain paraissent correspondre à la fortification d'une des collines marquant le paysage. Tout au long du Moyen Age ct durant l'époque moderne, les ruines de l'ancienne cité du Haut-Empire sont occupées ct transformées, maintenant sur Boismorcau une certainc vic citadine. La présence du Meucon marque une limite franclic entre les deux zoncs urbanisćes simplement reliécs par un pont. Si lc quartier Saint Patern est ravagé à chaque conflit car il n'est pas défendu par des remparts, les périodes de paix et d'essor ćconomique permettent toujours de le remettre en état. L'attachement ancien des habitants envers ce secteur vannetais découle certainement de la présence d'une ćglise dédiće aux reliques de Saint Patern. Mais, au-delà de cet argument mystique, le souvenir et les vestiges d'une partic de l'agglomération originelle pcuvent ćgalement expliquer la place particulière conservéc par ce quartier. Les nombreux réaménagements qu'il a done subis laissent imaginer les bouleversements connus par les vestiges antiques. L'évolution de l'occupation au 10 rue de la Tannerie ne peut ĉtre isoléc de ce contexte géographique et historique bien particulicr si on vcut évaluer au micux la place des ćléments découverts dans le phćnomène urbain. 


\section{LES ÉTAPES DE L'OCCUPATION SUR LE SITE DU 10, RUE DE LA TANNERIE}

Le terrain concerné par notre intervention présentait des vestiges antiques fortement bouleversés par les aménagements de l'époque moderne. En dehors de quelques creusements perturbant notre vision en plan des structures, un terrassement de grande ampleur ne nous a laissé qu'une surface réduite de niveaux gallo-romains en place dans la moitié sud du terrain (environ $415 \mathrm{~m}^{2}$ ).

De ce côté, les installations les plus anciennes recoupent une épaisseur de terre brune qui recouvre naturellement le substrat granitique. Les quelques tessons présents dans la partie supérieure de ce niveau sont peut-être contemporains des travaux d'aménagement du site (abattage, débroussaillage, nivellement, etc.). On note une majorité de céramique non tournée accompagnée de fragments d'amphores du type Pascual I et Dressel 7/11. Quelques formes en terra nigra (Menez 96 et Menez 126) ont aussi été identifiées. L'ensemble indique une datation précoce pouvant remonter à la fin du Ier siècle av. J.-C.

LES PREMIĖRES INSTALLATIONS (Période 1 - Fin du Ier siècle av. J.-C. - Début du Ier siècle ap. J.-C.) (fig. 2)

\section{Une organisation primaire}

Parni les aménagements qui recoupent le terrain naturel, certains creusements linéaires présentent des orientations qui semblent obéir, de façon approximative, à un schéma commun. L'ensemble détermine en effet une ligne discontinue aménagée du nord-oucst au sud-est s'orientant vers le nord-est dans le bas du terrain. Toutes ces structures, au profil semi-circulaire peu profond ( $0,20 \mathrm{~m}$ en moyenne), matérialisent peut-être une limite entre deux espaces. Du côté nord, les dispositions autonomes de certaines structures isolées permettent d'imaginer des aménagements primaires contemporains, puisque tous les vestiges postérieurs respecteront des orientations communes parfaitement orthogonales. L'ensemble évoque une première organisation sommaire caractérisée par la construction de bâtiments légers mis en place dans des espaces grossièrement délimités. A ce stade, les caractéristiques d'une ville de tradition romaine (trame orthogonale, chaussées aménagées, etc.) n'apparaissent tonc pas dans l'emprise de la fouille.

\section{Les traces d'installations légères}

Malgré l'état fragmentaire des vestiges, la présence de constructions en bois se devine grâce à la découverte de structures particulières pouvant correspondre à des empreintes de poutres et de poteaux. Dans la partie haute du site, de telles installations recoupent un modeste sol de mortier directement aménagé sur le terrain naturel. Plus bas, une poutre carbonisée in situ peut être interprétée comme une sablière basse appartenant à une construction sur parois porteuses. L'élément en bois n'était que très légèrement enfoncé dans le sol. Il a simplement été déposé dans une tranchée profonde de 3 à $4 \mathrm{~cm}$ vraisemblablement destinée à le caler. L'épaisseur d'un sol pouvait masquer cette sablière basse même si aucune trace n'en était conservée.

\section{Abandon et datation}

Ces premières structures ont livré très peu de mobilier datant. Toutefois, la proportion importante de céramique non tournée, la présence de sigillée exclusivement italique (service Ic et service II) et l'identification de plusieurs for-

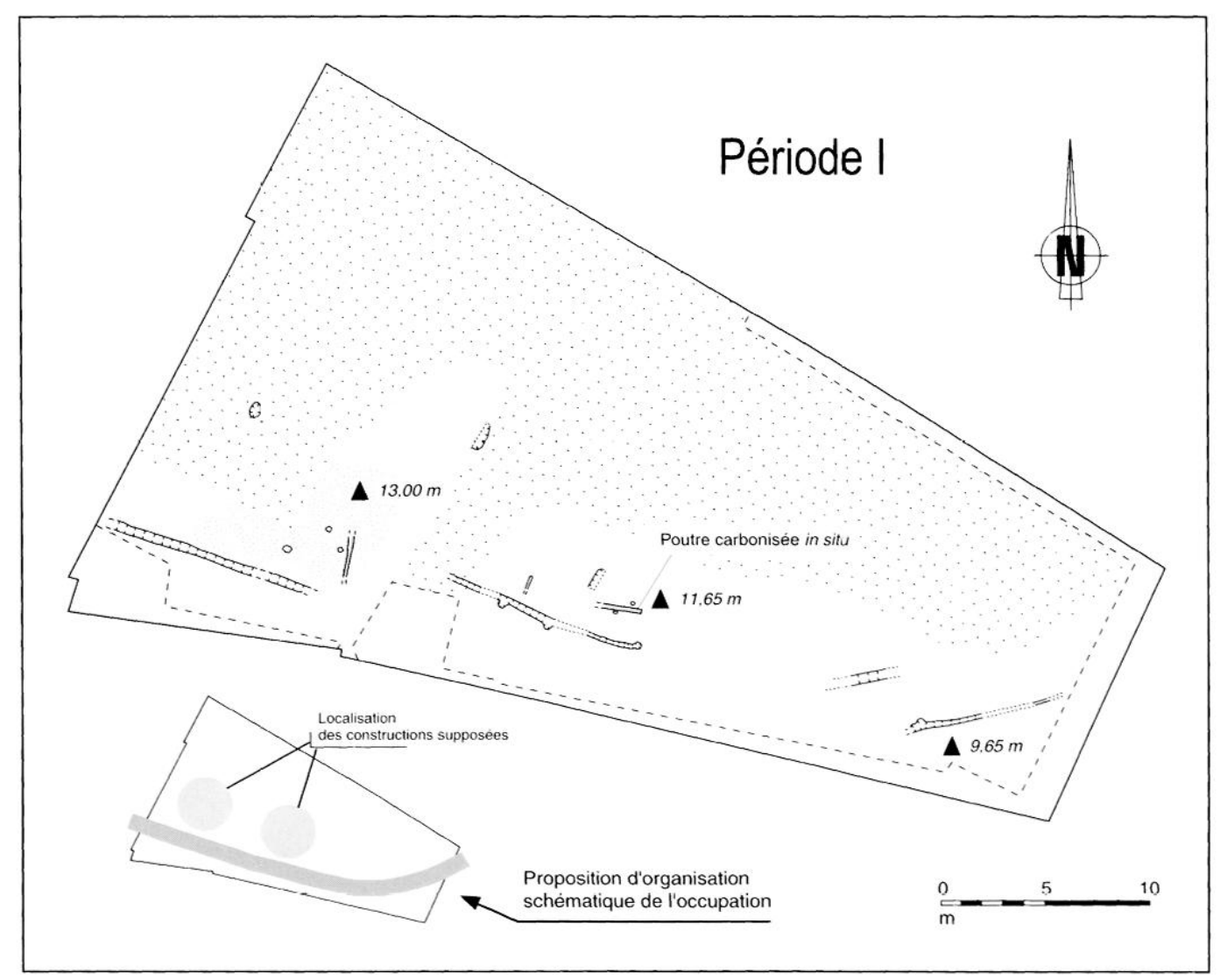

Fig. 2 : Plan des vestiges de la période 1. 
mes en terra nigra (Menez 22,125,126 et 128) permettent d'envisager une datation augustéenne. Deux fibules du type Feugère 14a (Feugère, 1985, 262-267), également mises au jour dans ces niveaux anciens, viennent conforter cette cstimation puisqu'elles sont bien typiques de ces horizons chronologiques (fig. 3). La fouille de la grande tranchée située à l'ouest a pourtant livré un fragment de sigillée du sud de la Gaule qui ramènerait son comblement aux années 15-20 de notre ère. Il est toutefois possible que ce tesson appartienne au remblai, riche en mobilier, qui scelle ce remplissage et qui s'est affaissé dans la tranchéc.

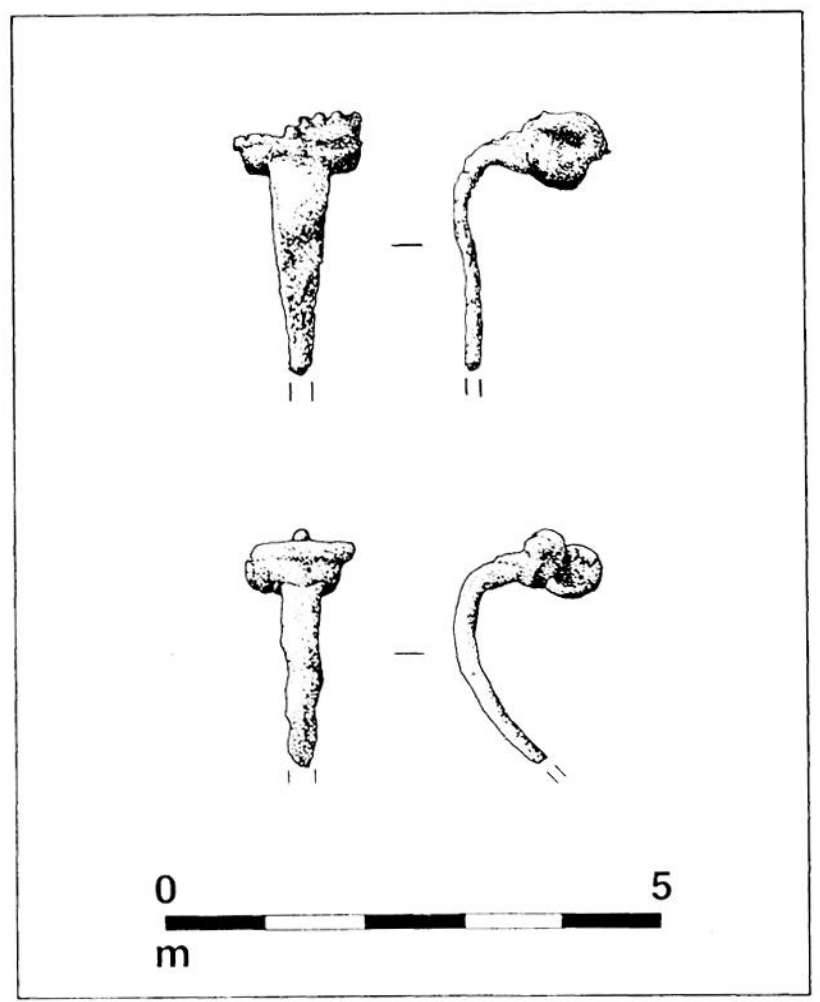

Fig. 3 : Fibules type Feugire 14 a retrouvées dans les niveaux d'abandon de la période 1.

L'URBANISATION DU QUARTIER (Période 2 - Première moitié du Ier siècle ap. J.-C.) (fig. 4 à 8 )

La mise en place de l'urbanisme correspond à une phase relativement complexe où plusieurs installations évoluent de manière indépendante selon une organisation qui s'affirme peu à peu. Dans certains cas, l'absence de relation stratigraphique peut être compensée par un mobilier datant qui apporte parfois des indices essentiels et décisifs. Une évolution logique pcut alors ĉtre proposée pour l'ensemble du site. Mais, si chacun des aménagements évolue bien tout au long de cette période, tous ne sont pas forcement modifiés d'un état à l'autre.

\section{L'affirmation d'orientations dominantes}

Parmi les premiers creusements qui traversent le substrat, certaines structures respectent une organisation qui s'accorde déjà avec les orientations que suivra l'ensemble monumental qui sera construit au nord (catalogue Vannes, 1992, 29-34 et 92-96). Certains de ces aménagements recoupent des structures antćrieures, contrairement aux installations de la première période. Cette observation s'accorde bien avec une restructuration de l'espace dans un sccond temps (période 2a). Dans cet ensemble, une nouvelle limitc soulignće par une sćrie de tranchées marque le terrain du nord-ouest au sud-est. Cette fois, les aménagements contemporains apparaissent essentiellement du côté sud de cette ligne (fig. 4) mais l'important terrassement moderne qui a détruit toute la moitié nord du site a pu faire disparaître d'éventuelles structures placées de ce côté. De nouvelles constructions légères remplacent ou complètent alors les installations antérieures. Rien ne montre, en effet, que la mise en place de cette nouvelle disposition du quartier implique la destruction systématique de tous les aménagements de la période 1 . Au contraire, l'organisation semble s'affirmer peu à peu selon le nouveau schéma qui, lui, n'est pas forcement marqué au sol dans un premier temps. De simples bornes pouvaient, par exemple, indiquer les intersections entre les lignes majeures de ce dispositif.

La mise en place d'une zone de circulation orientée nordest/sud-ouest illustre parfaitement cette idée d'une transformation progressive. En effet, nous avons pu constater que les vestiges de la période 1 placés au centre du terrain sont recouverts, dans un second temps seulement (période $2 b$ ), par une fine épaisseur de cailloutis très compacte. Les traces rectilignes qui en marquent la surface y révèlent de nombreux passages de véhicules. Les nouvelles orientations établies précédemment sont ainsi confirmées par l'ćtablissement d'une zone de circulation orientóe nord-sud (fig. 5). Elle ne peut effectivement être établie avant la période $2 \mathrm{~b}$ car elle est strictement contemporaine d'un niveau de terre argileuse aménagé à l'est et qui, lui, recouvre des vestiges déjà alignés en fonction des orientations dominantes. Dorénavant le terrain se trouve clairement organisé en plusieurs parties : l'aménagement de la zone de circulation concrétise d'abord une séparation nette entre deux îlots (insulae) ; chacun d'entre eux paraît luimême subdivisé par une limite placée sur un axe commun perpendiculaire à la rue. Il est difficile d'établir le rôle exact de ces divisions internes qui se devinent seulement dans l'agencement des structures. Dans certains cas, elles correspondent peut-être à des limites de propriétés à moins qu'elles ne renforcent simplement les repères d'un quadrillage urbain à l'intérieur des îlots. A ce stade, de telles hypotheses sur la division de l'espace sont quand même à retenir avec une certaine prudence en raison du bouleversement important subi par la moitié nord du terrain

\section{La construction et l'évolution d'un bâtiment en terre et bois}

Dans le bas du terrain, deux tranchées perpendiculaircs sont disposées selon la nouvelle organisation dès la période $2 \mathrm{a}$ (fig. 4). Un tel plan peut correspondre à des empreintes de sablières se poursuivant au-delà des limites est et sud du chantier. Dans ce cas, les deux tranchées désigneraient l'angle nord d'une construction en bois (ensemble A). Mais la présence de céramique dans leur comblement indique que le bâtiment aurait été complètement démonté jusqu'aux poutres qui supportaient les parois. Le mobilier recueilli présente une forte proportion de céramique non tournée associée à des productions caractéristiques de la période augustéenne (fig. 9).

L'absence de relation stratigraphique nous oblige à envisager la conservation de l'ensemble A au moment de la mise en place de la première zone de circulation établie au milieu du terrain (période $2 b$, fig. 5). Ce n'est qu'en période $2 c$ que le bâtiment $B$ aurait été construit sur les vestiges de l'ensemble A (qui n'en constituait probablement qu'un premier état relativement simple) (fig. 6). L'organisation du 


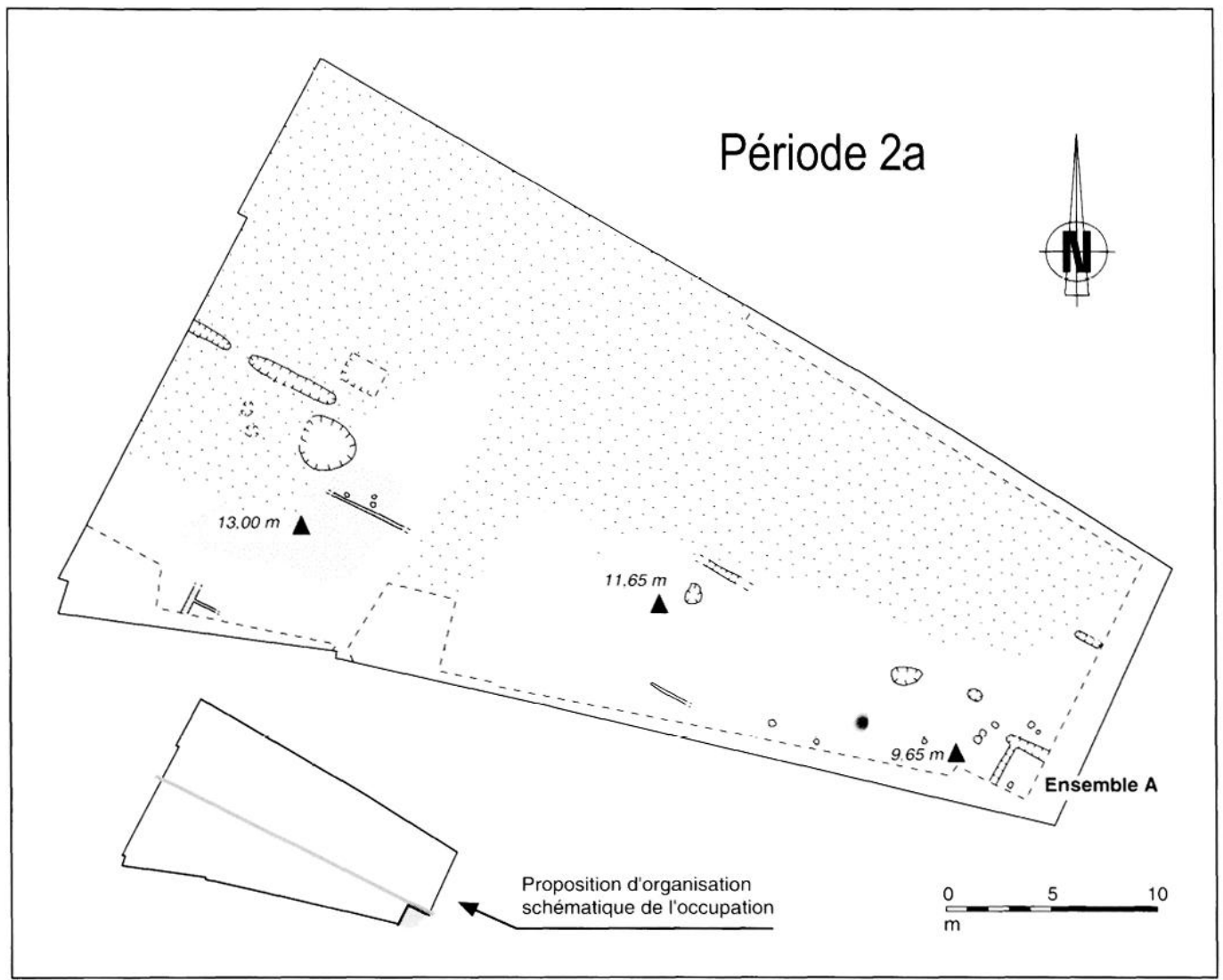

Fig. 4 : Plan des vestiges de la période 2 a.

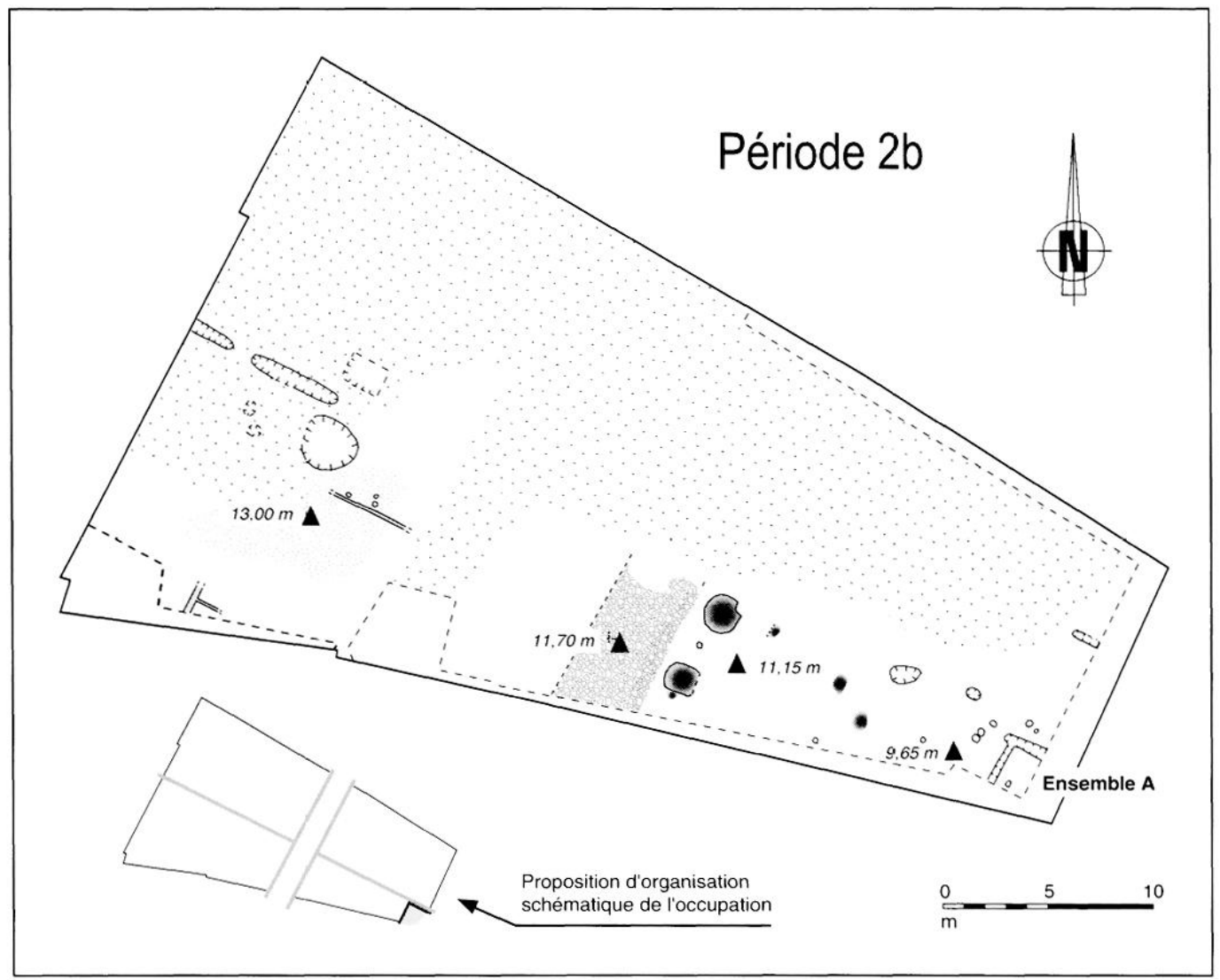

Fig. 5 : Plan des vestiges de la période $2 b$. 


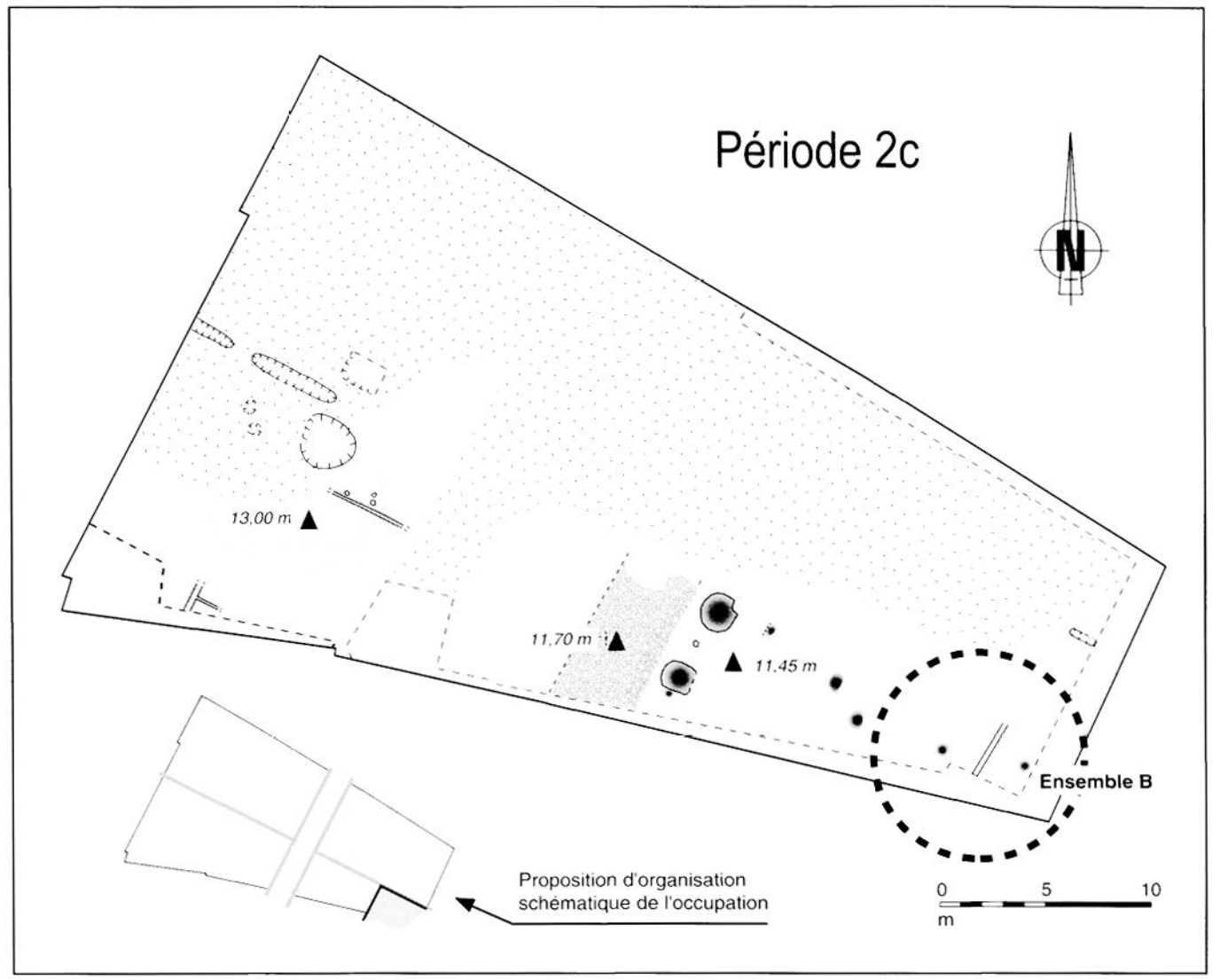

Fig. 6 : Plan des vestiges de la période 2 c.

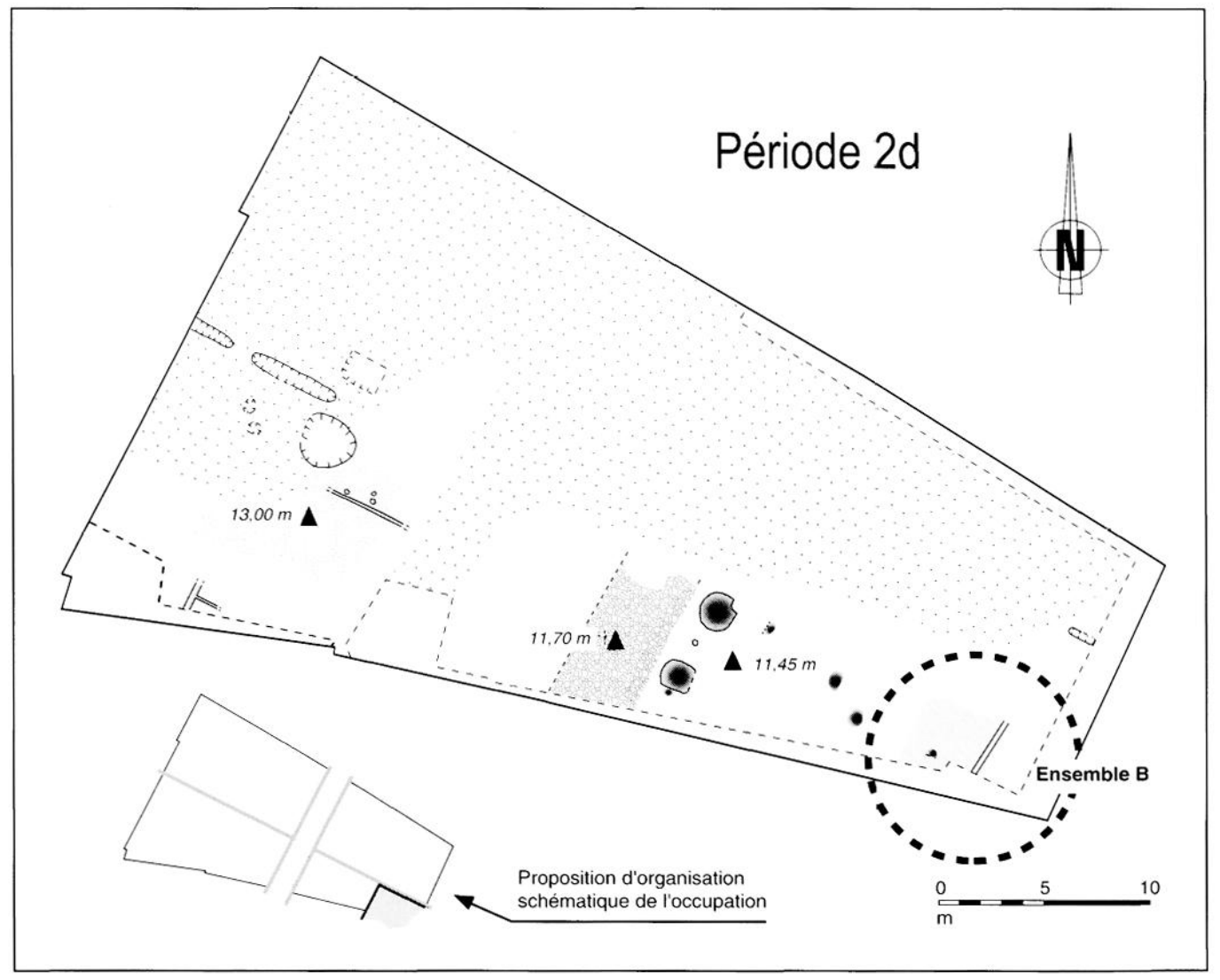

Fig. 7 : Plan des vestiges de la période $2 \mathrm{~d}$. 


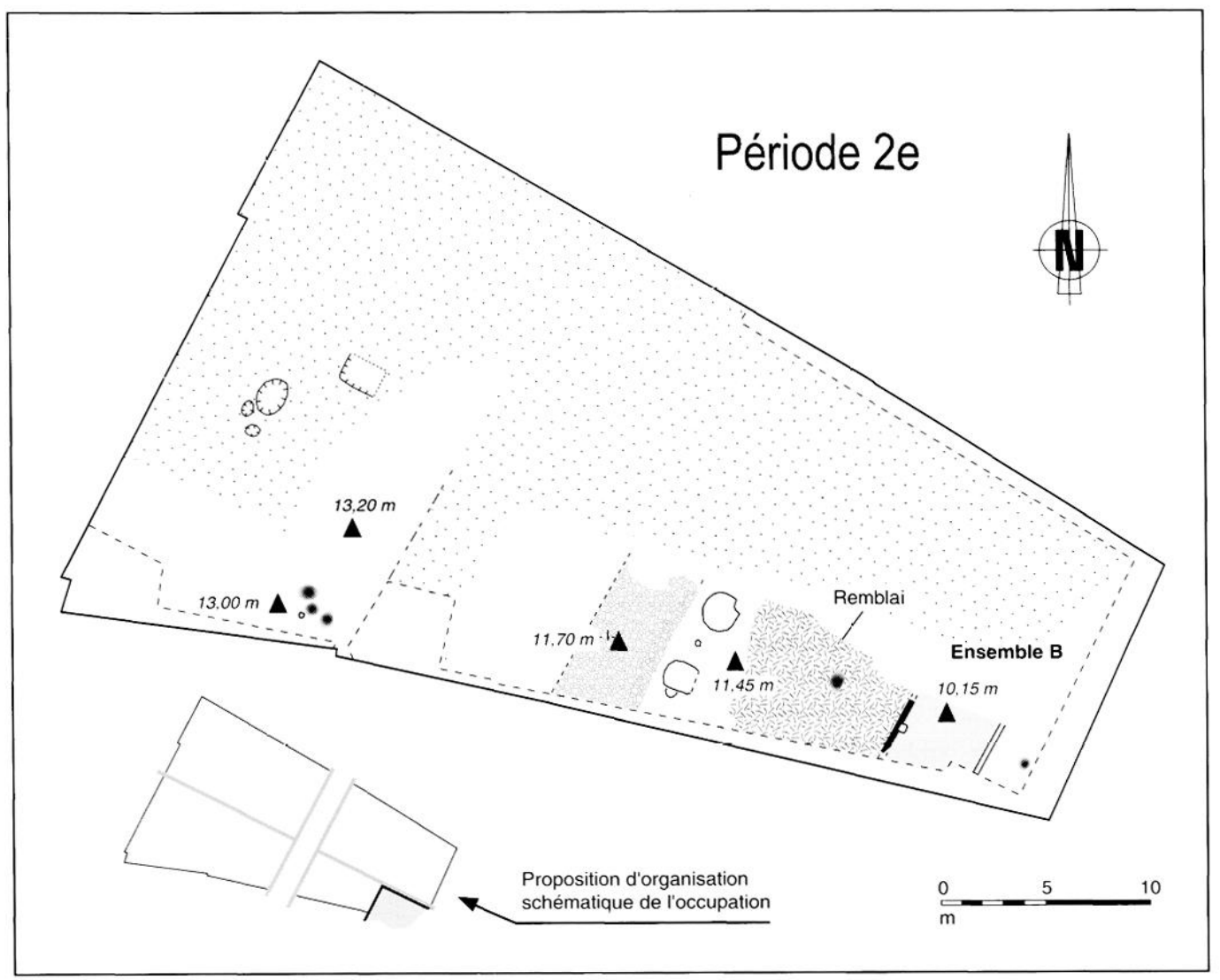

Fig. 8 : Plan des vestiges de la période $2 e$.

nouveau bâtiment apparaît à travers les restes de sols qui révèlent par endroits certaines empreintes de cloisons en bois. Une coupe stratigraphique à travers ces niveaux montre clairement la méthode de construction employée (fig. 10). Deux pièces quadrangulaires sont ainsi séparées par une paroi qui s'appuic sur une poutre sablière basse orientée du nord au sud. De part et d'autre, un radier de blocs de pierres sert de soubassement à des niveaux de mortier dont les surfaces ont été damćes. La sablière basse. posée au préalable, est donc masquée par l'épaisseur des sols qui lui sont associés. Dans la pièce placée du côté ouest. le mortier se prolonge au-delà de l'emprise du radier. Il est donc possible que les blocs soient surtout destinés à égaliser le niveau à l'emplacement d'un creusement antérieur éventuellement lié à l'ensemble A. Ce dernier pourrait rappeler, dans ce cas, certaines constructions cxcavées de tradition indigène tclles qu'on en connait au village de Goulvars à Quiberon. aux Ebihens à St Jacut de La Mer ou sur le site de Polvern à Hennebont (Menez. Daire, Hyvert, Langouët, Lc Bihan et Tanguy, 1990, 129. fig. 8 et 9). Comme tous ces bâtiments se rapportent à une période tardive de l'époque gauloise (Ier s. av. J.-C.), le remplacement de notre ensemble $A$ par la construction $B$ pourrait illustrer un aspect particulier de l'évolution des techniques architecturales au début de la période romaine.

La pièce occidentale du bâtiment $B$ a subi deux agrandisscments successifs (périodes $2 \mathrm{~d}$ et 2 c) révélés par l'aménagement de nouveaux sols qui ćlèvent le niveau (fig. 10). De ce fait, la différence de hautcur qui cxistait déjà entre Ics sols des deux pic̀ces, est passéc de $10 \mathrm{~cm}$ à $20 \mathrm{~cm}$. impliquant la préscnce d'une marche encore plus marquéc en cas de passage de l'unc à l'autrc. La première réfection est recoupce par l'amćnagement d'un mur de terrasse destiné à soutcnir un remblai installé à l'ouest (période 2e, fig. 8). Cette ćpaisscur de terre brunc, qui recouvre l'espace resté libre entre le bâtiment $B$ et la zone de circulation, a livré quelques fragments de céramique. Outre la présence d'un tesson de sigillée de Montans type Drag. 27, la découverte d'un fragment de pot ovoïde à engobe sablé permet de dater ce remblai d'une période postérieure à $30 \mathrm{ap}$. J.-C. Par extension, la construction du mur de terrasse ne peut être antérieure à cette époque. Un petit puisard a été prévu sous la maçonnerie afin de récupérer les eaux de ruissellement. Son ouverture se distingue à la surface du dernier sol retrouvé dans la pièce occidentale du bâtiment B (fig. 10, état 3). Ainsi, la construction d'une terrasse affirme une nouvelle fois l'organisation urbaine déterminée précédemment puisqu'elle la respecte. Elle permet de niveler l'espace de travail situé entre la zone de circulation et le bâtiment $B$ facilitant ainsi les activités qui s'y développent. Enfin, l'évacuation des eaux qui apparaît dans l'aménagement du puisard peut également répondre à un souci de protection du bâtiment $B$.

Les vestiges de l'ensemble $B$ sont scellés par une épaisseur de terre argileuse de couleur jaune. La partie supérieure de ce niveau est soulignée par des fragments d'enduit blanc. L'ensemble peut correspondre à des parois en terre effondrées sur place. Les quelques tessons piégés dans ce niveau s'inscrivent dans une fourchette chronologique qui va des annécs 40 à la fin du Ier siècle. Outre la découverte d'un tesson en terra nigra type Menez 119, la présence d'une estampille rétrograde sur une coupelle de La Graufesenque indique parfaitement ce contexte chronologique. En effet, la marque PRIMI qui s'y lit est connue à Mctz sur une forme indéterminće associée à un ensemble daté entre 50 et 75 ap. J.-C (Deru \& Feller, 1996). Notons cnfin la découverte d'un fragment de sigillée type Drag. 35-36 du centre qui pourrait ramener la datation de ce remblai jusqu'au début du IIe siècle. Mais les nombreuscs fosses qui le recoupent permettent d'envisager ici l'intru- 
sion d'un ćlément appartenant à unc phase postéricure. C'est donc plus par honnêteté scientifique que par réclle conviction sur sa pertinence que nous signalons ce tesson.

\section{Le développement d'activités artisanales}

Un niveau argilcux placé à l'est de la zone de circulation recouvre les vestiges de la période 2 a (fig. 5). Sa surface daméc correspond à un sol strictement contemporain de la voic. Deux petits foycrs y sont directement installćs. L'un d'entre cux est recoupé par une fosse, d'un diamètre de $1,50 \mathrm{~m}$, au fond de laquelle plusicurs foyers se sont succédés. Une installation identique a ćté retrouvćc un peu plus au nord à une même distance de l'espace de circulation. Ces structures de combustion excavćcs sont remblayćes par une terre limoncuse brune qui a livré du mobilier augustcen (voir plus loin).

Dans le haut du terrain, le sol de morticr, aménagé dès la période 1, est recouvert (progressivement ?) par un niveau de terre noire dans lequel sont mélangés des rejets domestiques divers (huîtres, céramiques, clc.). Une grande fosse creusće légèrement au nord cst combléc par ce même dépotoir qui s'étend sur une surface d'au moins $50 \mathrm{~m}^{2}$. Ce niveau, qui se poursuit vers le sud au-delà de la limite de fouille, a livré beaucoup de mobilicr. L'essenticl se rattache aux annécs 30-40 ap. J.-C. Sans qu'aucun ćlément de l'enscmble ne paraisse antéricur à cette ćpoque (fig. 11). On notera nćanmoins que la proportion de céramique non tournéc reste encore importante sans toutcfois ĉtre majoritaire. Ce contexte chronologique cst conforté par la découverte d'une monnaic de Tibère "a l'autel de Lyon". Par ailleurs, la présence proportionnellement importante de grands récipients (dolia, amphores, gros vases, etc.) rappclle la découverte d'ćventucls rejets d'un entrepôt. rue du four, dans des horizons chronologiques ćquivalents (catalogue Vannes. 1992, 88-89). Ici encorc. ces possibles ćléments de conservation ou de stockage pourraient ćvoquer la proximitć du port. Ce remblai est ensuite niv clé pour l'amćnagement d'un sol en argile jaune sur lequel trois foyers sont installés (fig. 8). A proximitć, une petite cuvette est creuséc dans le niveau d'occupation. Tapissé d'unc ćpaisscur d'argile qui a chauffé. cet aménagement ćtait comblé d'une terre charbonncuse dans laquelle des particules de fer et de bronze ont ćté retrouvćes. La découverte d'un fragment de creuset dans l'épaisscur d'un des foyers est un indice supplémentaire pour reconnaître ici les vestiges d'un petit atclier de bronzicr. Une fosse retrouvée un peu plus au nord est peut-ĉtre liće à cet ensemble bien que l'absence de relation stratigraphique nous interdise de l'affirmer. Son comblement ćtait pourtant composé de rcjets de foycrs mélangćs à une terre très charbonneuse dans laquelle des éléments en bronze ont aussi été retrouvés. Une anse de bassin y avait même été rejetée (fig. 12). Elle appartient à un modèle reconnu à Colchester dans des contextes datćs du milicu du Ier siècle de notre c̀re (Crummy. 1983, 71-73, n²039 et 2044). Le mobilier associé comprenait des fragments de rćcipients en sigillće de Montans type Drag. $15-17$ et Drag. 17 ainsi qu'un tesson de sigillée du centre de la Gaule type Ritt. 5. L'ensemble ne contredit nullement une appartenance éventuclle de cette fosse à la période $2 \mathrm{c}$.

Deux zones de travail sont donc ćtablies de part et d'autre de l'axc de circulation. Celle qui se place du côté est peut fonctionner à partir de la pćriode 2 b alors que la partic haute du terrain sern:ait de dépotoir. La seconde ne semble mise en place qu à la fin de la période 2 . A ce titre, clle succède peut-ĉtre à la premic̀re dont les comblements des fosses-foyers contenaicnt un mobilicr attribuable à la fin du règne d'Auguste au plus tôt (sigillíc italique de service II. sigilléc du sud de la Gaule types Irag. 17 et Drag. 19, terra nigra types $1 / e^{\prime \prime n e} 222$. 64 et 96 . céramique non tournćc).

\section{A la recherche de precisions chronologiques}

Le mobilier associć à l'abandon de la pćriode 1 et celui retrouvé dans les derniers nivcaux lićs à la période 2 permettent de situer la mise en place de cette dernic̀re phase entre le règne d'Auguste et la scconde partie du ler sic̀cle ap. J.-C. Les fluctuations chronologiques inhérentes aux durées d'utilisation des formes céramiques ne permettent guc̀re plus de précision pour l'instant. Cette période. d'une cinquantaine d'annóes environ. voit se succéder cinq ćtats qu'on peut pourtant essayer de situer plus prćcisćment les uns par rapport aux autres.
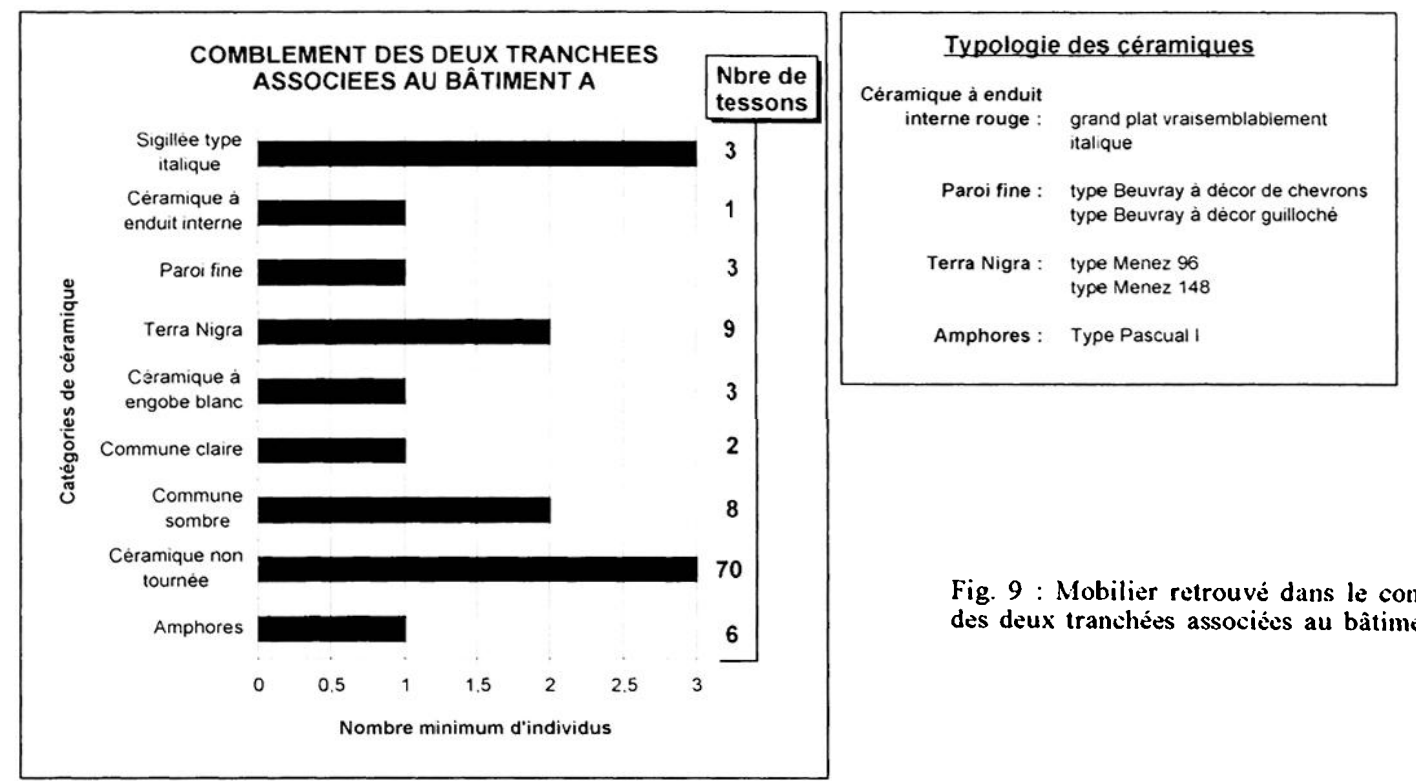

Fig. 9 : Mobilier retrouvé dans le comblement des deux tranchées associćes au bâtiment $A$. 


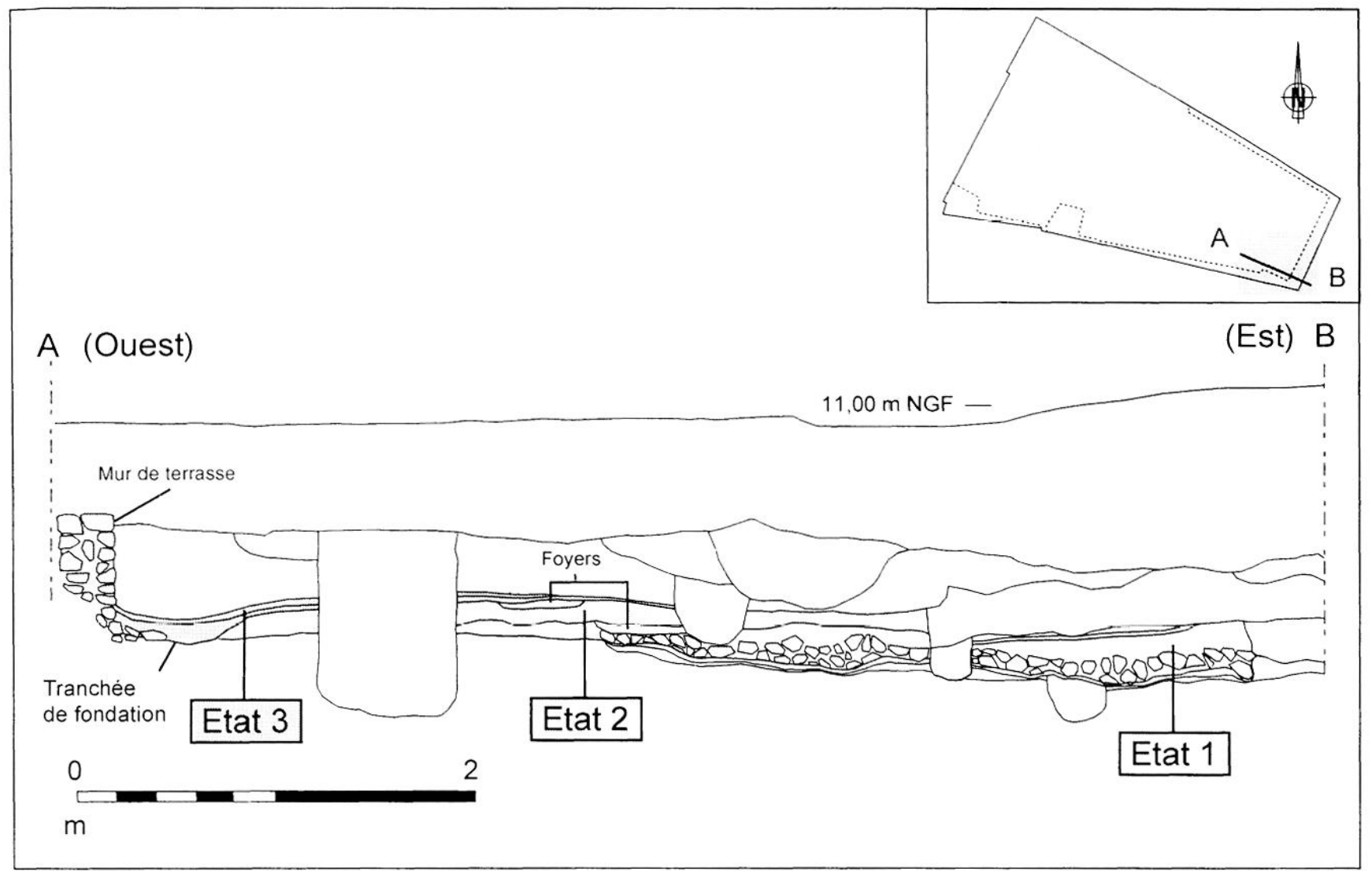

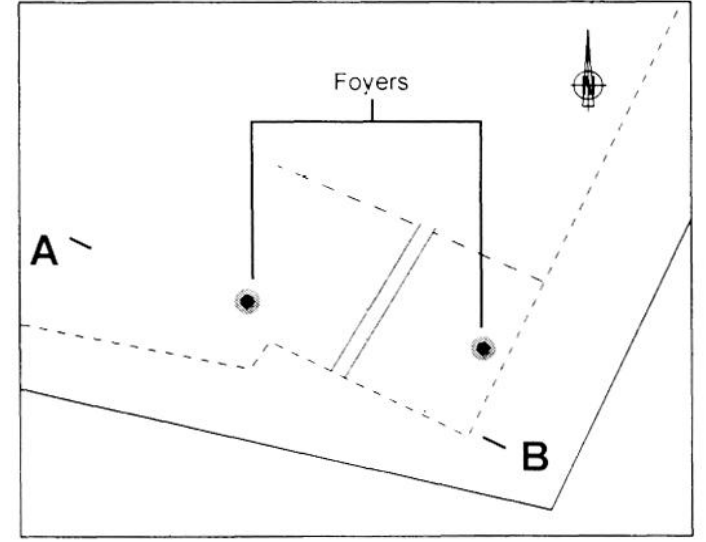

Etat 1 (période 2c)

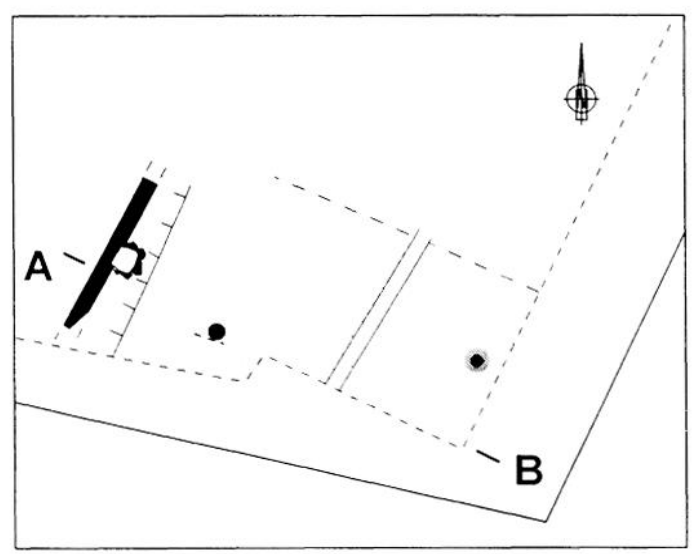

Tranchée de fondation du mur de terrasse

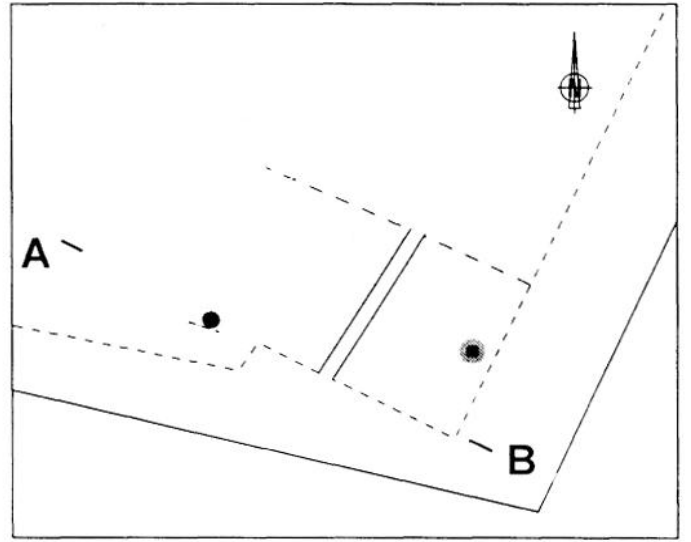

Etat 2 (période 2d)

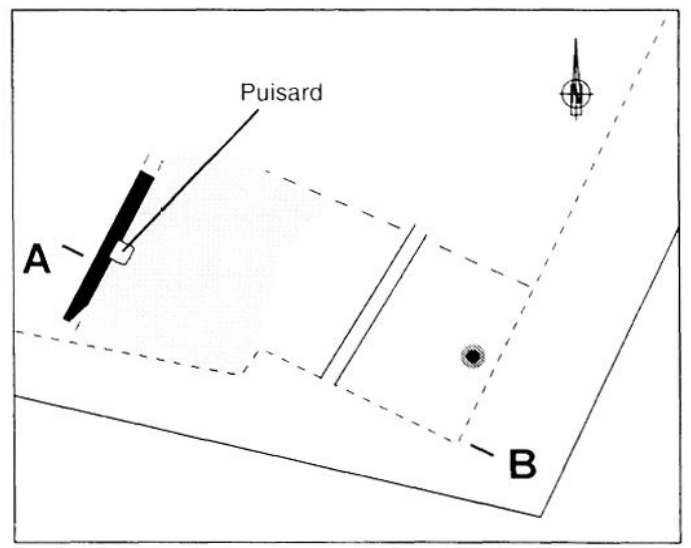

Etat 3 (période 2e) 


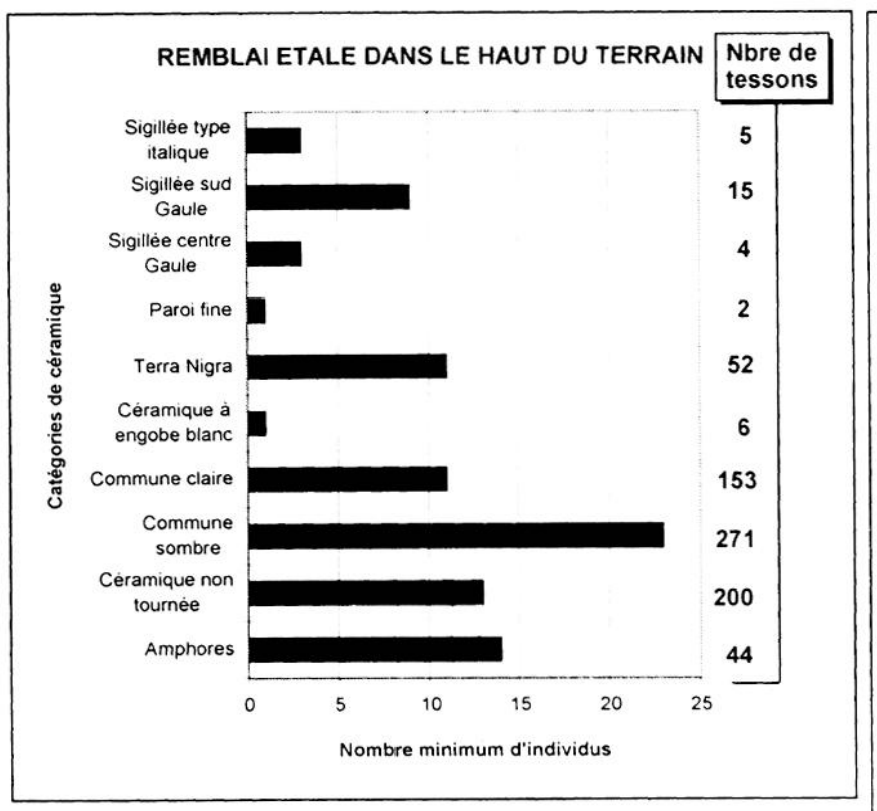

Fig. 11 : Mobilier retrouvé dans le remblai étalé dans le haut du terrain en période 2.

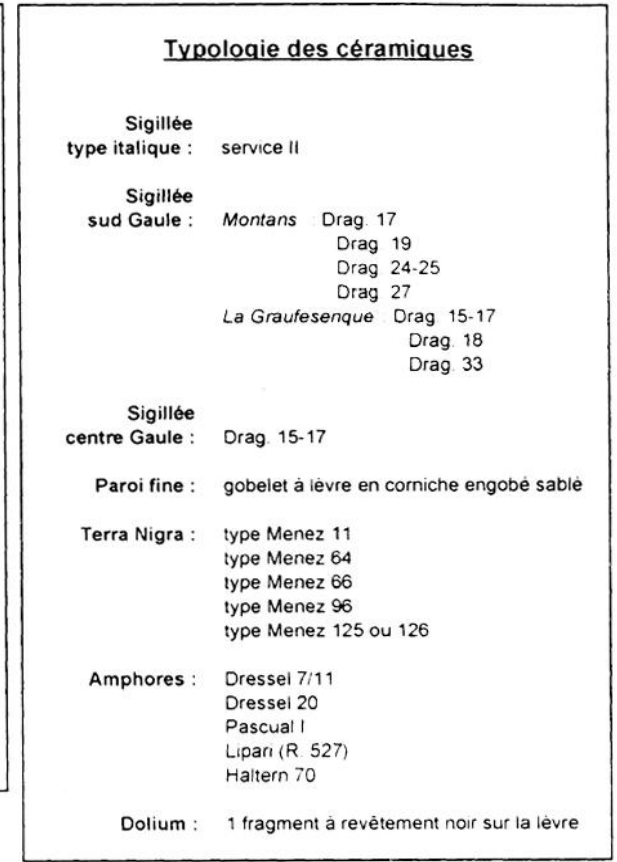

Les aménagements des périodes $2 \mathrm{a}$ ct $2 \mathrm{~b}$ n'ont pas livré d'éléments assurément postéricurs au règne d'Auguste. Au contraire, la proportion importante de sigillćc italique dans les niveaux d'abandon s'accorde avec une occupation limitce à l'époque augustćenne. L'absence de sigillćc de production gauloise renforce également cette idéc.

Par contre, les aménagements de la période $2 \mathrm{e}$ sont nécessairement établis vers 30 de notre ère au plus tôt. Un laps de temps d'une quinzaine d'annécs sćpare donc les états $2 b$ et $2 e$. Il suffit largement à permettre la construction du bâtiment $B$ dont l'aspect en période $2 \mathrm{e}$ est l'aboutissement d'une évolution en 3 étapes ( $2 c, 2 d$ et $2 c)$. Les périodes $2 c$ et $2 d$ pourraient donc s'inscrire dans la première partie du règne de Tibère (entre 15 et 30 ap. J.-C).

Enfin, la période 2e doit au moins s'étendre sur une vingtaine d'années au vu du mobilier du milieu du Ier sic̀cle retrouvé dans les niveaux de destruction.

Les caractères urbains s'affirment ici à travers les relations établies entre les différents ćléments du quarticr. Ils apparaissent ainsi dans l'aménagement du site qui confirme l'implantation sur unc organisation préalablement établic et scrupulcusement respectéc. Par aillcurs, le remplacement du bâtiment A par l'enscmble B indique unc adaptation au relief par l'aménagement d'une petite terrasse. Cettc évolution peut être liće au développement urbain du quartier qui modèle pcu à peu le paysagc. La romanisation transparaît alors dans l'aménagement du sectcur et l'ćtablissement de l'urbanisme dont l'affirmation passe par l'évolution technique des constructions. De même, les ensembles artisanaux qui apparaissent le long de l'axe de circulation illustrent les activités que suscite l'épanouissement d'une ville naissante. Les indices chronologiques montrent enfin que la romanisation qui se développe sous Auguste pourrait s'aflirmer, de manic̀re encore plus fortc. à partir du règne de Tibère.

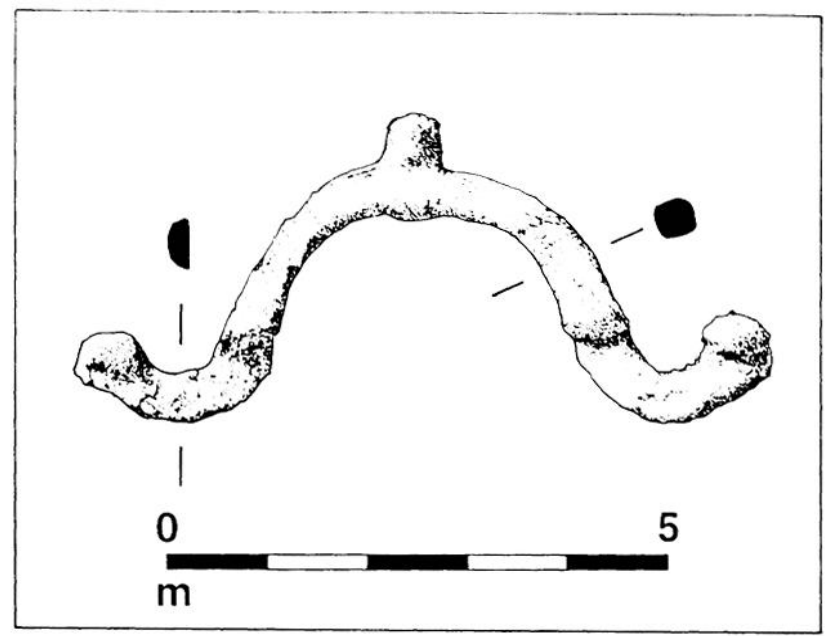

Fig. 12 : Anse de bassin en bronze.

STRUCTURES PONCTUELLES OU PRÉMICES D'UN ESSOR URBAIN ? (Période 3 - Milicu du Ier siècle ap. J.-C. / 3e quart du Ier sic̀cle ap. J.-C.) (fig. 13).

\section{Affirmation et recadrage de l'organisation de l'espace}

Deux fossés perpendiculaires recoupent les niveaux d'abandon de la pćriode 2. Ils affirment encore une fois les orientations choisies précédemment.

Le premier est parallèle à l'axe de circulation retrouvé au milicu du sitc. II se place à 2 in de son bord est et recoupe lc comblement des deux fosses-foycrs de l'état antérieur. La surface plane et compacte des remplissages indique bien qu'on a circulé sur les remblais de ces structures. Cette observation révèle l'ćlargissement de la voirie, probablement motivce par l'augmentation du trafic qui accompagne le développement urbain.

L'autre fossé a été retrouvé dans la partie est du terrain où il recoupe le niveau de terre qui scelle l'ensemble B. Les aménagements qui ont suivi ne permettent pas d'évaluer la longucur totale du creusement. 


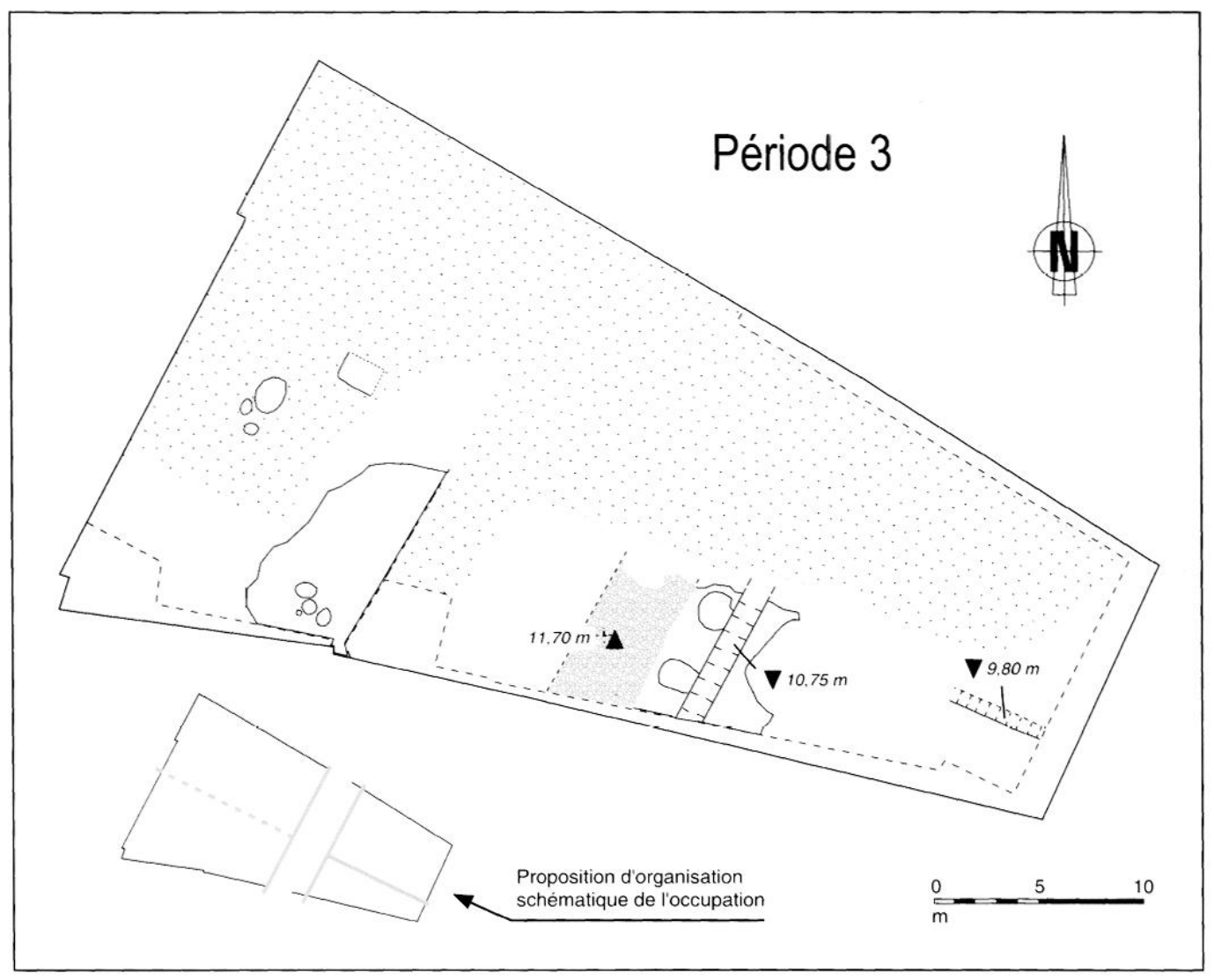

Fig. 13 : Plan des vestiges de la période 3.

Il paraît important de signaler ici la découverte d'un autre fossé lors d'interventions ponctuelles réalisées par $\mathrm{A}$. Triste dans des terrains situés au nord de la rue de la Tannerie. Selon le fouilleur, c'est effectivement une même structure qui aurait été mise au jour au 11 rue de la Tannerie et dans la parcelle qui forme l'angle du boulevard de la paix et de la rue de l'étang (renseignement A. Triste). Or l'alignement de ces deux points correspond exactement à l'axe d'un gros mur qui sera établi sur notre site au cours de la période suivante (fig. 14). La présence d'un troisième fossé sous cette maçonnerie est donc à envisager pour la période 3 .

\section{Les indices d'une phase de transition}

Aucune construction nouvclle n'est associée à cette ćtape de l'occupation dans l'cmprise que nous avons fouillće. L'établissement d'un simple réseau de fossés paraît en constituer la seule caractéristique. Pourtant, si la destruction de l'ensemble B est prouvće, rien ne permet de savoir si l'artisanat qui s'est développé en haut du terrain est toujours en activité. Enfin, la zone de circulation semble alors utilisée car son extension a ćté damée. Mais l'absence de traces d'ornic̀res ne s'accorde pas avec une existence très longue de cet état de voirie. En outre, sa fragilité aurait dû permettre d'en marquer d'autant plus facilement la surface. Si la volonté d'établir pour longtemps une zone de circulation avait donc ćtć mise en ocuvre à ce moment, il est évident que la construction d'une chaussée plus résistante aurait étć entreprise.

Il semble qu'on ait sculement voulu recadrer l'organisation urbaine en prévision d'une nouvelle phase de développement. Alors que le creusement du bas confirme une limite cntre deux lots à l'intćricur d'un même îlot, l'ćtablissement d'un fossé le long de l'axe de circulation marque

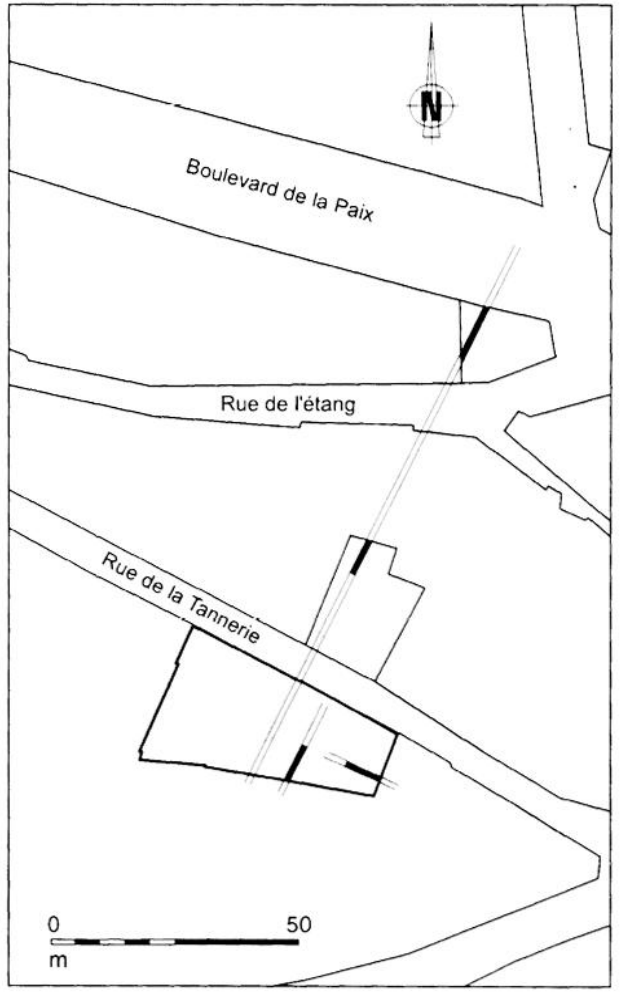

Fig. 14 : Mise en relation du réseau de fossés de la période 3 avec des sections de fossés fouillées par $A$. Triste.

clairement un élargissement de l'espace public.

Les comblements des deux fossés renforcent l'idće d'une phase chronologiquement limitéc. Sur ce point l'absence de trace de colluvionnement dans le fond montre d'abord qu'ils n'ont pas été ouverts très longtemps mais l'aspect 
homogène de chacun des remplissages indique également un remblaiement rapide.

\section{Eléments de datation}

Le creusement longeant la voie ne contenait qu'une terre limoneuse marron alors que la fouille de la seconde structure a livré une quantité importante de mobilier (fig. 15). Dans ce lot, l'ensemble des céramiques sigillées, désormais dominé par les productions du sud de la Gaule, contient quelques exemplaires du centre. Dans un tel contexte, les formes italiques encore présentes sont nécessairement résiduelles. Cette ćvolution est renforcée par une diminution sensible des formes non-tournées qui constituaient encore le type majoritaire dans certains ensembles de la période 2 . Les transformations socio-économiques qui apparaissent ici pourraient se rattacher aux règnes de Claude ou de Néron puisque la datation de ce lot de mobilier semble essentiellement couvrir les années 40-60 ap. J.-C.

\section{LE DÉVELOPPEMENT DE L'URBANISME (Période 4 -} deuxième moitić du Ier siòcle ap. J.-C.) (fig. 16).

\section{Renforcement du cadre urbain}

Unc épaisscur de cailloutis mélangé à du morticr extrêmement compact scelle la zone de circulation établie en période $2 b$. Cette nouvelle chaussće recouvre également une grande partie de l'espace de travail aménagé à l'est de la voirie initiale. Elle s'atténue vers l'est dans le fossé creusé en période 3 .

Deux nouvelles constructions sont mises en place de part et d'autre de la zone de circulation. L'hypothèse d'une limite est-ouest supposéc dès la pćriode 2 a est alors confortéc par l'alignement des bâtiments sur cet axe précis. Quelques blocs de pierres dans des tranchées de fondation particllement disparues en constituent les seuls vestiges. Dans les deux cas. la partic nord des constructions a été complètement détruite par les terrassements postérieurs.

\section{Les premières constructions maçonnées}

Le bâtiment élevé à l'est (ensemble C) n'apparaît plus aujourd'hui qu'à travers trois murs établis sur un simple plan quadrangulaire. L'édifice aménagé à l'ouest (ensemble D) présente, quant à lui, un plan bien particulier dans lequel trois pièces allongées sont placées côte à côte. La salle centrale est légèrement plus large. Les perturbations modernes ne permettent pas d'assurer que l'essentiel du bâtiment apparaît ici. En effet, on peut également penser que cette disposition tripartite s'intègre à une construction plus vaste se prolongcant vers le nord-est. Des aménagements plus légers pouvaient même se rattacher à cet ensemble dans l'emprise fouillée ici. Mais, l'arasement du terrain est si important que des murs peu fondés ou des installations en bois trop légères ont pu disparaître sans laisser aucune trace. De plus, l'important degré de destruction de l'édifice, dont les murs ont été récupérés jusqu'aux fondations, invite encore plus à la prudence et rend bien hasardeuse toutc interprétation sur la nature de l'occupation.

\section{Arguments chronologiques}

Le mobilier associé à l'abandon des structures de la période 3 permet de concevoir la construction des premiers bâtiments maçonnés dans la seconde moitié du Ier siècle au plus tôt. Sur la fouille de la Z.A.C. de l'étang, localisée directement au nord, les fouilleurs conçoivent l'arrivée de la maçonnerie dès le deuxième quart du Ier siècle (catalogue Vannes 1992, 94). L'étude approfondie des résultats de cette opération devrait fournir des arguments chronologiques mieux définis et plus développés. Mais, pour l'instant, rien ne permet d'assurer la coexistence de ces vestiges avec les éléments de la période 4 définie au 10, rue de la Tanncrie.

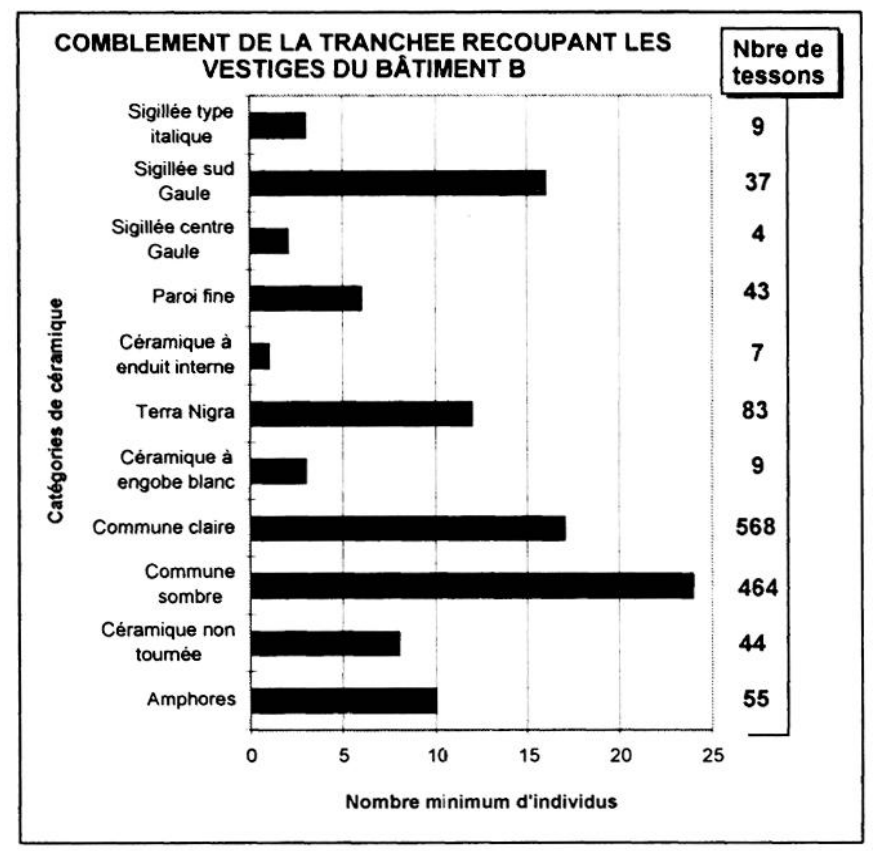

Fig. 15 : Mobilier retrouvé dans le comblement de la tranchée qui recoupe les vestiges du bâtiment $B$.

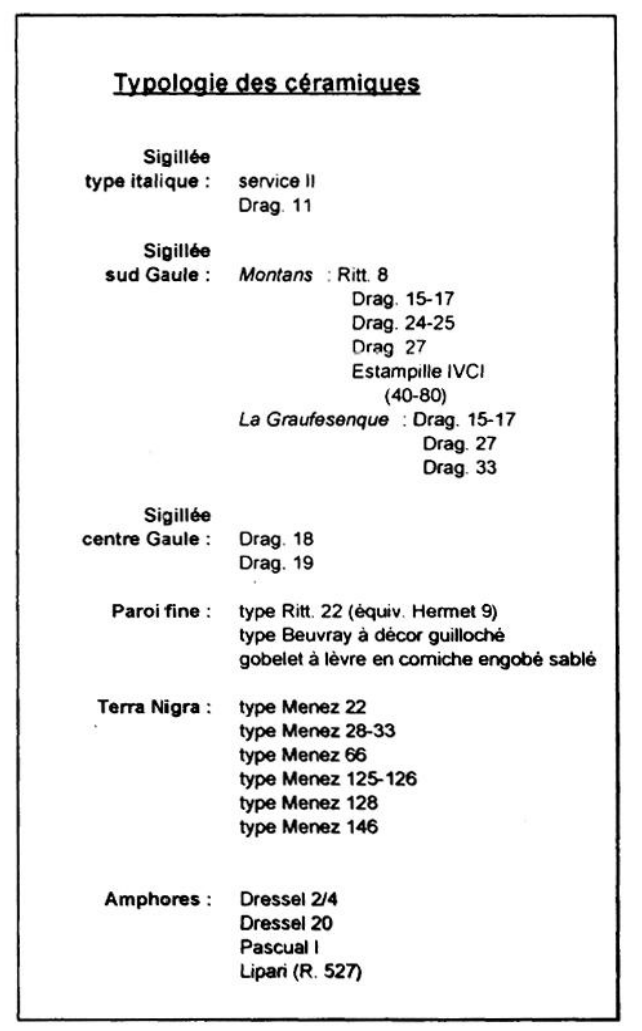




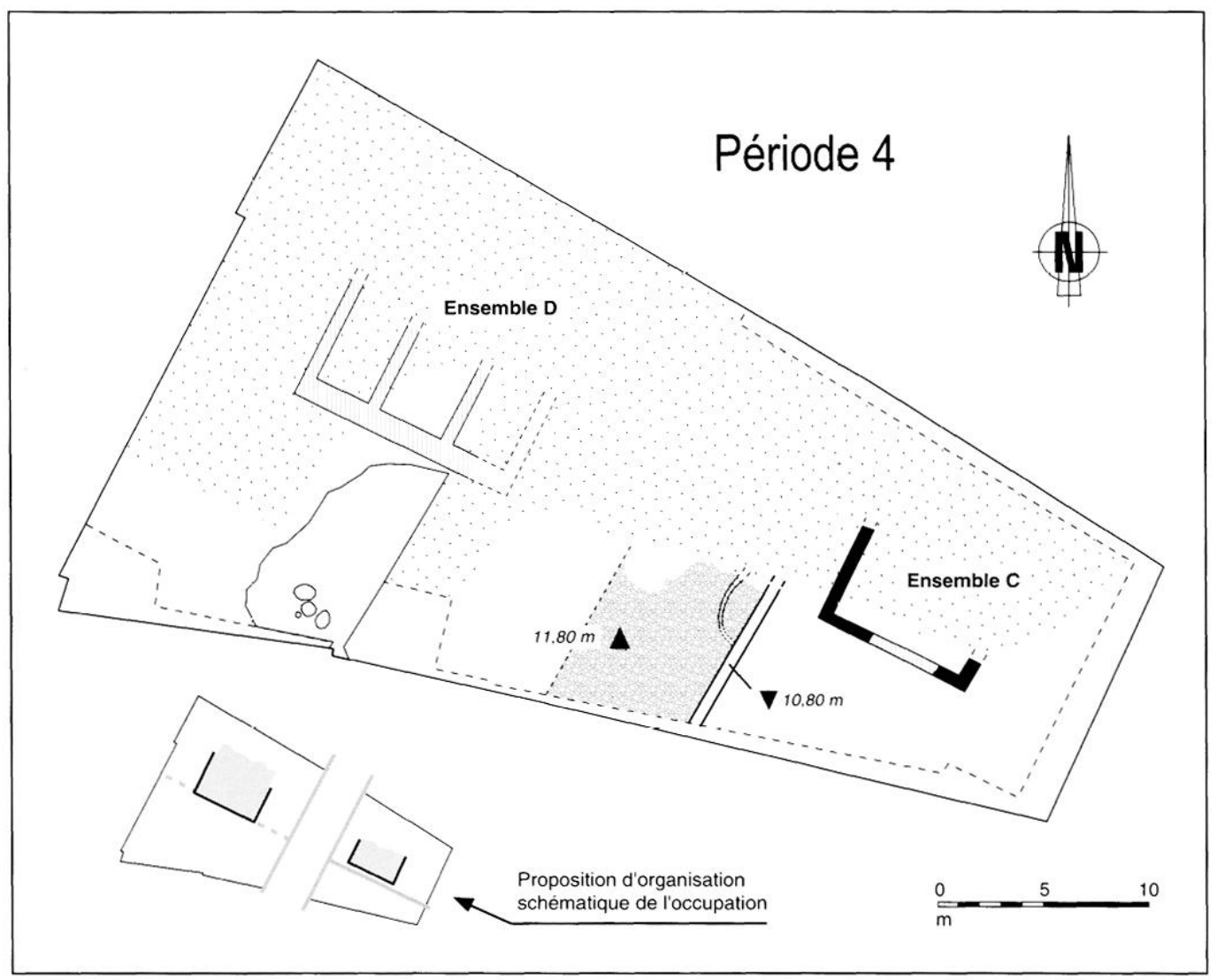

Fig. 16 : Plan des vestiges de la période 4.

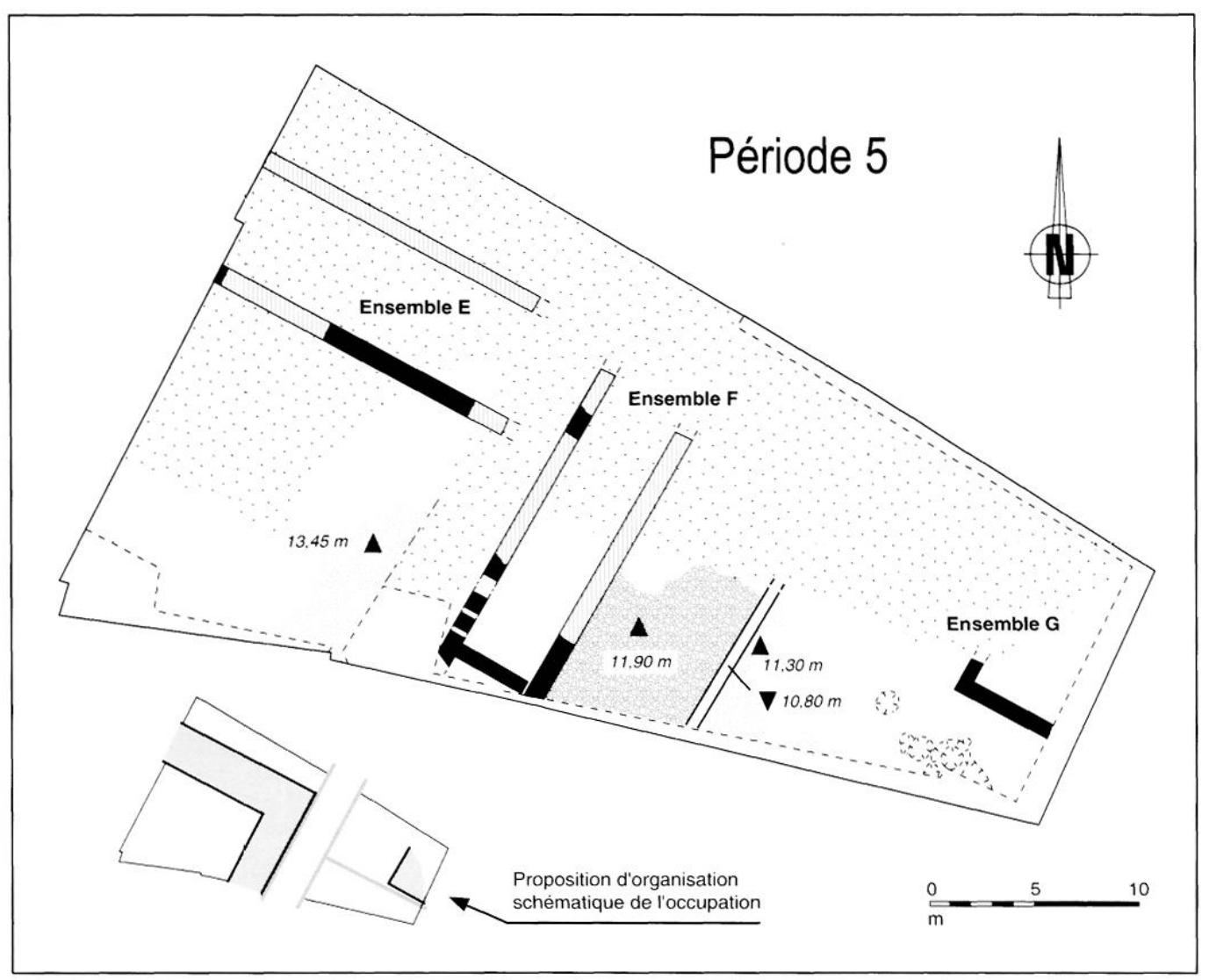

Fig. 17 : Plan des vestiges de la période 5. 
LA MONUMENTALISATION DE L'ARCHITECTURE (Période 5 - Fin du ler siècle ap. J.-C. - IIIle siòclc ap. J.-C.) (fig. 17).

\section{Maintien et disparition de certaines limites}

L'emprise de la zone de circulation redéfinie en période 3 n'est plus remise en cause. La protection de cet axe résulte vraisemblablement de son caractère public et révèle l'existence d'une autorité capable de l'assurer. Par contre, si la limite qui divise l'îlot est sert toujours de référence, les deux parcelles qui se trouvent à l'ouest sont réunies dans un même ensemble par la construction d'un complexe nouveau. Il semble d'ailleurs possible que ce rapprochement parcellaire ait étć rćalisć dès la période 3 même si l'architecture ne le rend réellement évident qu'à ce stade du développement urbain.

\section{La construction d'un ensemble imposant à l'ouest}

Le bâtiment $D$ est détruit pour laisser place à un ensemble architectural relativement imposant composé de deux galeries perpendiculaires aménagées à des niveaux différents (ensembles $E$ et $F$ ). Les inurs, fondés sur la roche saine, présentent une maçonncric extrêmement résistante. D'une largeur de $0,90 \mathrm{~m}$. les fondations sont constitućes de radiers en gros blocs de granit sur lesquels plusieurs lits de pierres liées au mortier se succèdent jusqu'au niveau de construction. Seul un modeste vestige d'élévation a été retrouvé. Il présentait encore deux assises de petits moellons parfaitement ordonnćs en parement alors que le blocage interne était essentiellement composé de mortier. La nature de ce dernier correspondait à celui des fondations. Sa résistance résulte d'une teneur en chaux très importante qui lui confère une couleur blanche bien caractéristique. La recherche de solidité est donc évidente. Elle témoigne d'un bâtiment imposant et élevé dont la technique de construction est comparable à celle de l'ensemble monumental fouillé au nord (catalogue Vannes, 1992, 30-34 et 94-96). Une épaisscur de morticr jaune très compacte a ćté retrouvée sur une surface de $10 \mathrm{~m}^{2}$ situće entre les deux corps de bâtiment. Ce niveau, qui scelle le sol de travail de la période $2 e$ sur $0,20 \mathrm{~m}$ d'épaisseur, pourrait fonctionner avec la grande construction qui l'encadre car son importance et son homogénéité s'accordent avec l'ampleur d'un tel projet architectural. On pourrait alors y voir les vestiges d'un sol limité, à l'origine, par les deux galeries.

Mais cet enscmble peut faire partie d'un programme encore plus vaste auquel appartiendrait ćgalement la réfection de l'axe de circulation qui traverse le terrain. Ce dernier est effectivement revêtu d'une épaisseur de morticr très compacte qui vient buter contre le mur de la galerie $F$. Les fondations légèrement débordantes de la construction sont ainsi masquées (fig. 18). De grosses pierres posées à la surface du mortier confèrent une cohésion certaine à la chaussée et lui assurent en même temps une importance particulière. Cette recherche de qualité et de solidité peut répondre à un double souci de prestige et de résistance à une circulation intense. La volonté de réaliser un ouvrage qui dure transparaît dans tous ces éléments.

Parmi les rares tessons découverts dans l'épaisseur de cette chaussée, se trouvait un fragment de récipient type Drag. 37 produit à Montans entre la fin du Ier siècle et la première moitié du IIc siècle de notre ère.
Le fossé de bord de voie est probablement aménagé à cette époque. Un caniveau de section quadrangulaire y est installé. Un tel profil n'a pu être maintenu qu'à l'aide d'un coffrage en bois comme on en connaît à Lyon (rue des Farges et Verbe Incarnée) (Desbat, 1985, 78) ou à Oberwinterthur (Suisse) (Paunier, 1985, 118). Une coupe stratigraphique transversale montre que l'ensemble a été refait au moins une fois avant l'abandon du caniveau (fig. 18).

Le lien direct qui est établi ici entre la voie et l'ensemble architectural construit dans l'îlot ouest suggère l'intégration de ce dernier dans un aménagement public. Le nombre d'établissements de cet ordre disposant d'un système de grandes galcries périphériques est limité. Les palestres qui accompagnent les grands thermes publics représentent une comparaison possible. A Wroxeter (Grande Bretagne) le centre urbain est ainsi dominé par le forum accompagné d'un grand complexe thermal. Celui-ci est équipé d'une grande palestre formant la moitié de la surface de l'établissement (La Bedoyère (de), 1992, 81). A Limoges, le même schéma a pu être mis en évidence (Desbordes \& Loustaud. 1992, 113, 116, fig. 46, 47). A Trèves, les deux grands ensembles thermaux de la ville disposent d'aménagements comparables (Wightman, 1970, 83).

L'hypothèse d'un ensemble cultuel peut aussi être envisagée. En effet, de nombreux sanctuaires gallo-romains disposent de grandes galeries périphériques aussi imposantes que nos ensembles E et F : Cautaing-sur-Escaut, Meaux, Drevaut, Jublains, etc. (Fauduet, 1993, 39, 45, 51 et 61). L'isolement apparent de ces deux corps de bâtiment va également dans ce sens. La coexistence d'un grand ensemble cultuel à côté du forum est d'ailleurs connue à Glanum ou à Périgueux où le grand sanctuaire de la Tour de Vésone est construit au Ile siècle (Girardy-Caillat, 1992, 127, fig. 50).

Dans tous les cas, la mise en place de ces importantes galeries à des niveaux différents révèle l'établissement d'une terrasse conséquente à l'ouest de la voirie (fig. 19). L'ampleur des travaux suggère un aménagement public et souligne la transformation du paysage licé à l'affirmation de l'urbanisme.

Toutes ces idées confortent le rapprochement avec l'ensemble monumental fouillé au nord. La position de tous ces vestiges les uns par rapport aux autres peut alors être abordée sans incohérence notable. Au contraire, la place de chacun dans l'ensemble renforce la logique d'un plan préétabli (fig. 20). On s'aperçoit en effet que la voie retrouvée au 10 rue de la Tannerie est parallèle à la zone de circulation qui longe l'ensemble monumental à l'ouest. Or, le bâtiment identifié comme une basilique se place exactement entre ces deux axes dont l'écartement atteint $160 \mathrm{~m}$.

Ces observations permettent de soupçonner la mise en oeuvre d'un véritable projet architectural au sein d'un plan d'urbanisme tout à fait réfléchi. Le caractère vraisemblablement public des travaux expliquerait même la démolition complète de l'ensemble D situé sur l'emprise de ce " centre " monumental. La disposition de ces grands bâtiments confirme les orientations respectées par les aménagements successifs du quartier depuis la période 2. Cette partie de la ville antique de Vannes semble ainsi disposer d'une véritable trame orthogonale dont la mise en place pourrait remonter à cette époque. L'organisation et le rôle des constructions les unes par rapport aux autres restent néanmoins à déterminer. La place conséquente qu'elles occuperaient dans une cité estimée à seulement 40 hectares est un sujet qui demande également réflexion. 
Fig. 18 : Coupe restituée de la voirie, mettant en relation la chaussée avec son caniveau (les perturbations postérieures n'ont pas rendu possible la réalisation d'une coupe réelle de l'ensemble).
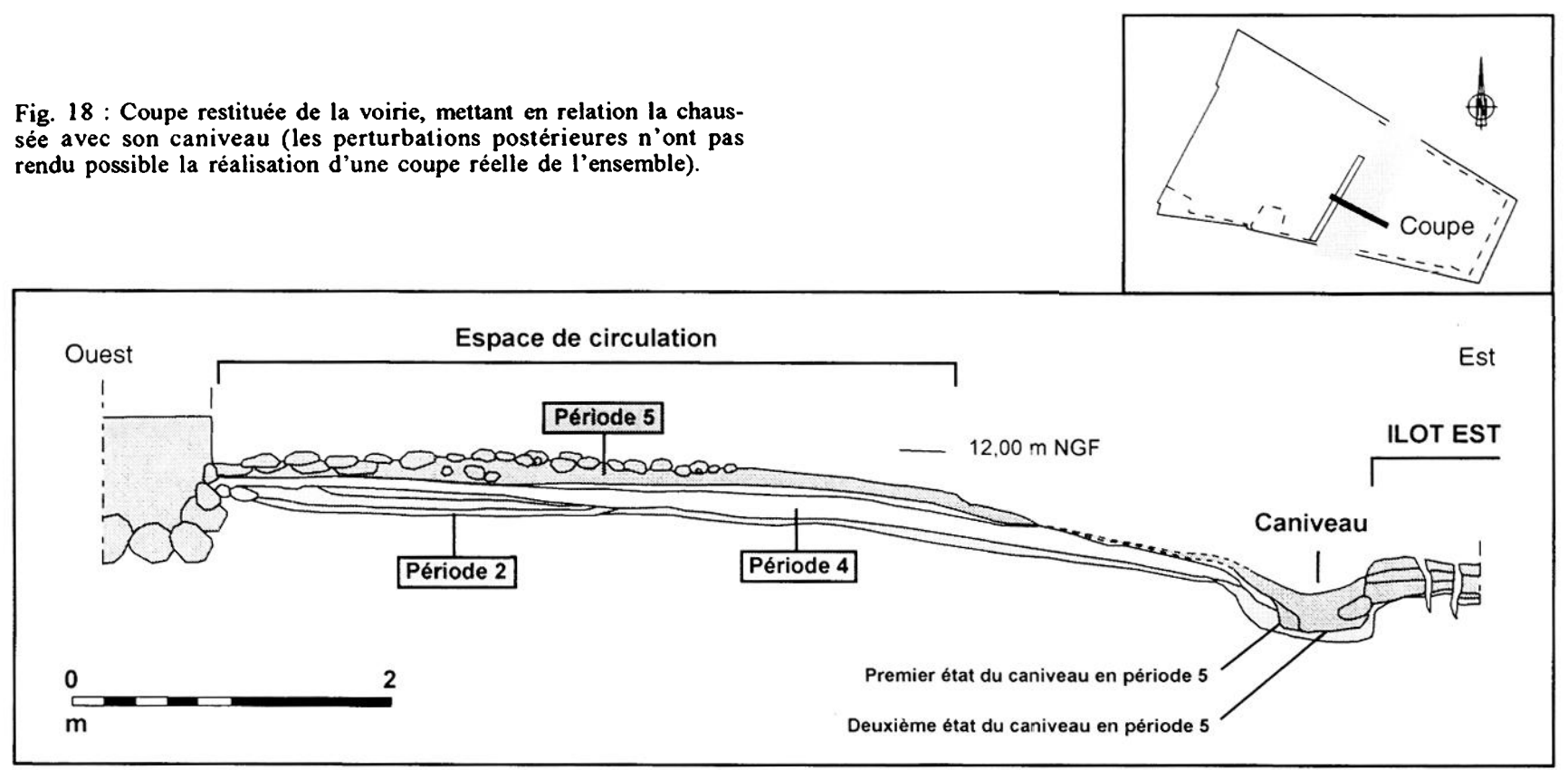

\section{Les nouveaux aménagements de l'îlot est}

La zone directement située à l'est du fossé est revêtue de plusieurs sols successifs en mortier. Leur alignement vertical révèle la présence d'une paroi en bois appartenant au coffrage du caniveau (fig. 18). Ces niveaux de circulation, de très bonne qualité, s'étirent vers le bas du terrain sur une longueur de $7 \mathrm{~m}$. Un terrassement postérieur les a fait disparaître au-delà de cette emprise.

Les vestiges d'une autre construction (ensemble $G$ ) ont été retrouvés dans la partie est du terrain. Seules deux maçonneries perpendiculaires étaient conservées. L'une d'elles, orientée du nord au sud, prend appui sur le radier d'un des murs du bâtiment $C$, préalablement rasé. Ces fondations extrêmement résistantes sont identiques à celles de l'ensemble $F$ et permettent d'envisager ainsi la coexistence des deux constructions, ce que ne contredit nullement la stratigraphie.

\section{Indices sur la durée de la période 5}

Très peu d'éléments apportent une information déterminante sur la durée de la période 5. L'indice le plus intéressant est constitué par la découverte d'une monnaie de Tétricus (3e quart du IIIe siècle) dans le niveau d'installation du dernier sol aménagé à l'est du caniveau de bord de voie. La qualité de cette réfection et le respect du système d'évacuation de l'eau indiquerait alors la persistance du cadre urbain établi en période 5 jusqu'à une période basse de l'époque gallo-romaine. Pourtant, la seule monnaie de Tétricus apparaît comme un indice bien faible pour confirmer cette hypothèse. L'absence de céramique appartenant réellement au Bas-Empire (sigillée d'Argonne, céramique à l'éponge, etc.) laisse également perplexe.

Fig. 19 : Essai de restitution du site en coupe au cours de la période 5 .

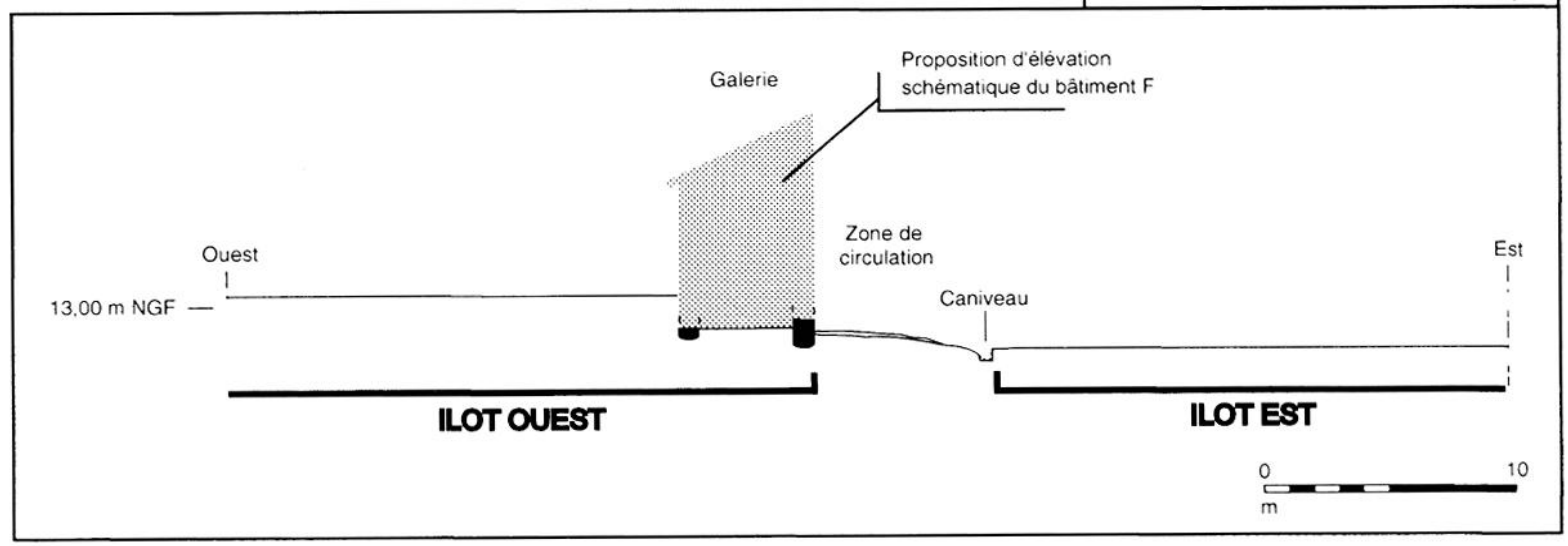




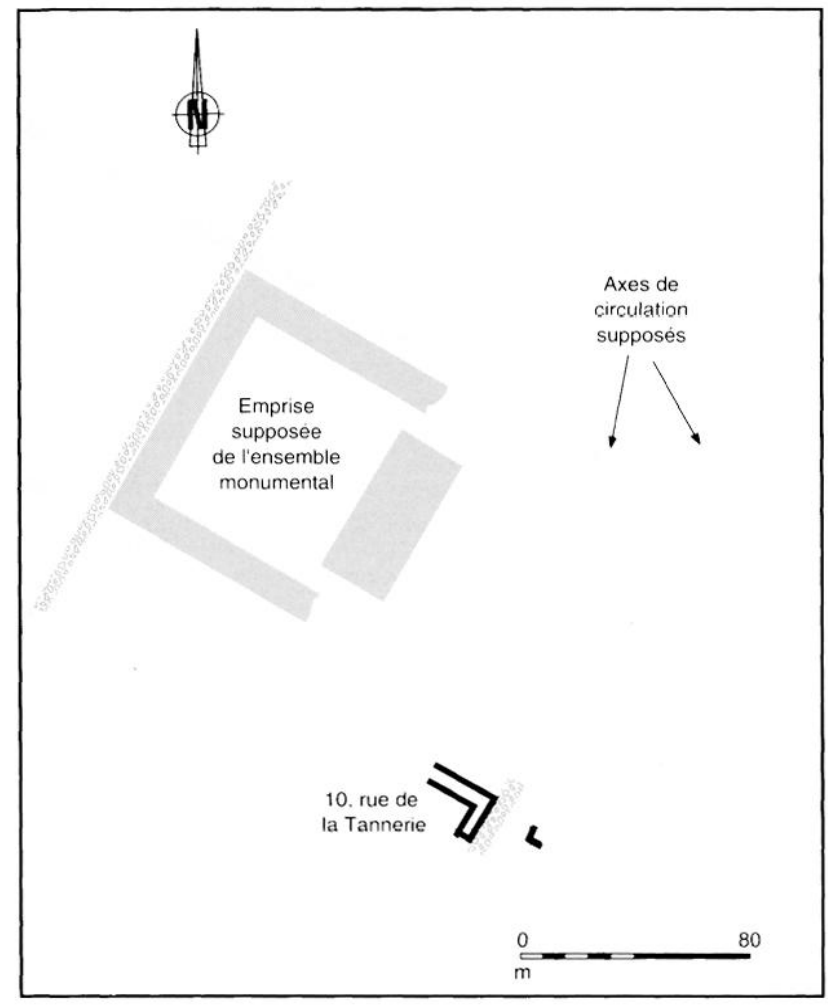

Fig. 20 : Proposition de mise en relation du site avec les vestiges monumentaux fouillés à proximité.

LA TRANSFORMATION DU QUARTIER (Période 6 Deuxième moitié du llle siècle ap. J.-C. ?) (fig. 21).

\section{Le respect du cadre établi}

Les mêmes orientations dominantes font toujours références lors des modifications du bâti qui marquent cette nouvelle étape de l'occupation. L'espace protégé que constitue la voie est encore respecté quand des constructions nouvelles sont établies. Quels que soient la nature ou l'état de ces dernières, les caractères essentiels de l'urbanisme du quartier existent donc toujours. On peut toutefois se demander si l'utilisation de ces repères correspond encore à la présence d'une autorité qui impose lcur respect, ou si des aspects pratiques de construction et de circulation n'incitent pas tout simplement les habitants de cette période à s'en servir. On remarque ainsi qu'aucun ćlément particulier ne permet d'estimer la division interne des îlots. Dans ce cas, une redistribution de l'espace privé est tout à fait possible alors que l'emprise du domaine public est maintenue.

\section{La médiocrité d'un nouveau bâti}

L'ensemble architectural mis en place au début de la période 5 est détruit. Les matériaux qui composaient les murs sont récupérés jusqu'aux fondations. Seule une partie de la maçonnerie qui longe la voie est conservée pour être intégrée à une nouvclle construction apparemment plus modeste (ensemble $\mathrm{H}$ ). En effet, un premier sol de terre battuc établi sur les vestiges de certains murs de la galerie F est recoupé par la construction d'unc nouvellc paroi fermant au nord ce qui subsiste du corps de bâtiment. Quatre tranchées parallèles ont été retrouvées à l'intérieur de cet espace d'au moins $32 \mathrm{~m}^{2}$. Orientées du nordouest au sud-est, elles présentent une largeur moyenne de
$0,20 \mathrm{~m}$. Une cinquième tranchée, perpendiculaire aux autres et légèrement plus fine, passe au milieu de la construction. L'ensemble évoque l'existence d'un plancher dont nous aurions ici les traces de solives. Une telle identification s'accorde parfaitement avec les observations habituelles dans ce genre de cas (Wacher, 1985, 150, fig. 10). L'importance des poutres peut néanmoins étonner. Mais les bouleversements importants du bâti et l'adaptation des nouveaux aménagements aux vestiges des ensembles précédents permettent d'imaginer toutes sortes d'arrangements. Dans un tel contexte, l'hypothèse d'une récupération de poutres de charpentes pour asseoir le plancher pourrait expliquer la démesure des empreintes.

Le caniveau de bord de voie est comblé par un remblai essentiellement composé de coquilles d'huîtres. Deux fosses distantes de $4 \mathrm{~m}$ recoupent ce niveau jusqu'au substrat. L'une d'elles présentait un comblement de blocs de pierres alors que la seconde ne contenait qu'une terre limoncuse marron. Le remplissage de la première correspond à un radicr de fondation qui supporte un bloc de calcaire quadrangulaire encore scellé par du mortier. On peut imaginer que la seconde structure était de même nature mais que l'enscmble de l'aménagement a été récupéré. Enfin, un radier très grossier relie les deux structures audessus de l'ancien caniveau. Il se poursuit vers le sud audelà de la zone fouilléc. Il devait également se prolonger vers le nord mais des récupćrations de matériaux et les terrassements modernes n'ont pas permis de l'observer clairement. L'ensemble, de facture maladroite, contraste fortement avec la rigucur architecturale du complexe précédent dont les orientations sont encore respectées ici.

Deux autres radicrs aussi sommaires sont mis en place un peu plus au sud. Bien qu'ils disparaissent dans la coupe qui marque la limite du chantier de ce côté et ne présentent ainsi que des bords approximativement roctilignes, ils semblent néanmoins perpendiculaires. Peut-être appartiennentils à un bâtiment relativement léger (ensemble I) dont nous n'aurions retrouvé ici que l'angle nord.

A proximité, plusieurs fosses recoupent le remblai qui marque l'abandon du bâtiment $B$ (période 2). Leur comblement, qui a livré du mobilier datable de la deuxième moitié du Ier siècle au début du IIe siècle (sigillée du sud de la Gaule type Drag. 22, Drag. 37) ne peut se rattacher qu'à la période 4 au plus tôt. Mais l'organisation particulièrement soignée des aménagements de cette période -et de la suivante- ne s'accorde guère avec la présence de tels creusements. Ces structures trouvent mieux leur place parmi les restructurations relativement grossières qui caractérisent la période 6.

\section{Entre Antiquité tardive et Moyen Age}

Si les ensembles de la période précédente sont bien entretenus jusqu'à la fin du IIIe siècle au moins, les restructurations importantes de la période 6 sont nécessairement postérieures à cette époque.

Rien ne permet pourtant d'y voir une occupation médiévale. Le respect de l'espace de circulation autant que le maintien de l'organisation antique sont des indices qui plaideraient plutôt pour une modification du paysage urbain de l'agglomération gallo-romaine sans qu'un bouleversement général des structures administratives en soit forcement la cause. La présence exclusive de céramique antique associóe aux nouvelles installations pourrait confirmer cette idće si le hiatus qui marque le Haut Moyen Age ne posait la question de la nature de l'occupation à cette époque. Mais, la découverte de cent pour cent de mobilier résiduel du Haut-Empire dans tous ces aménage- 


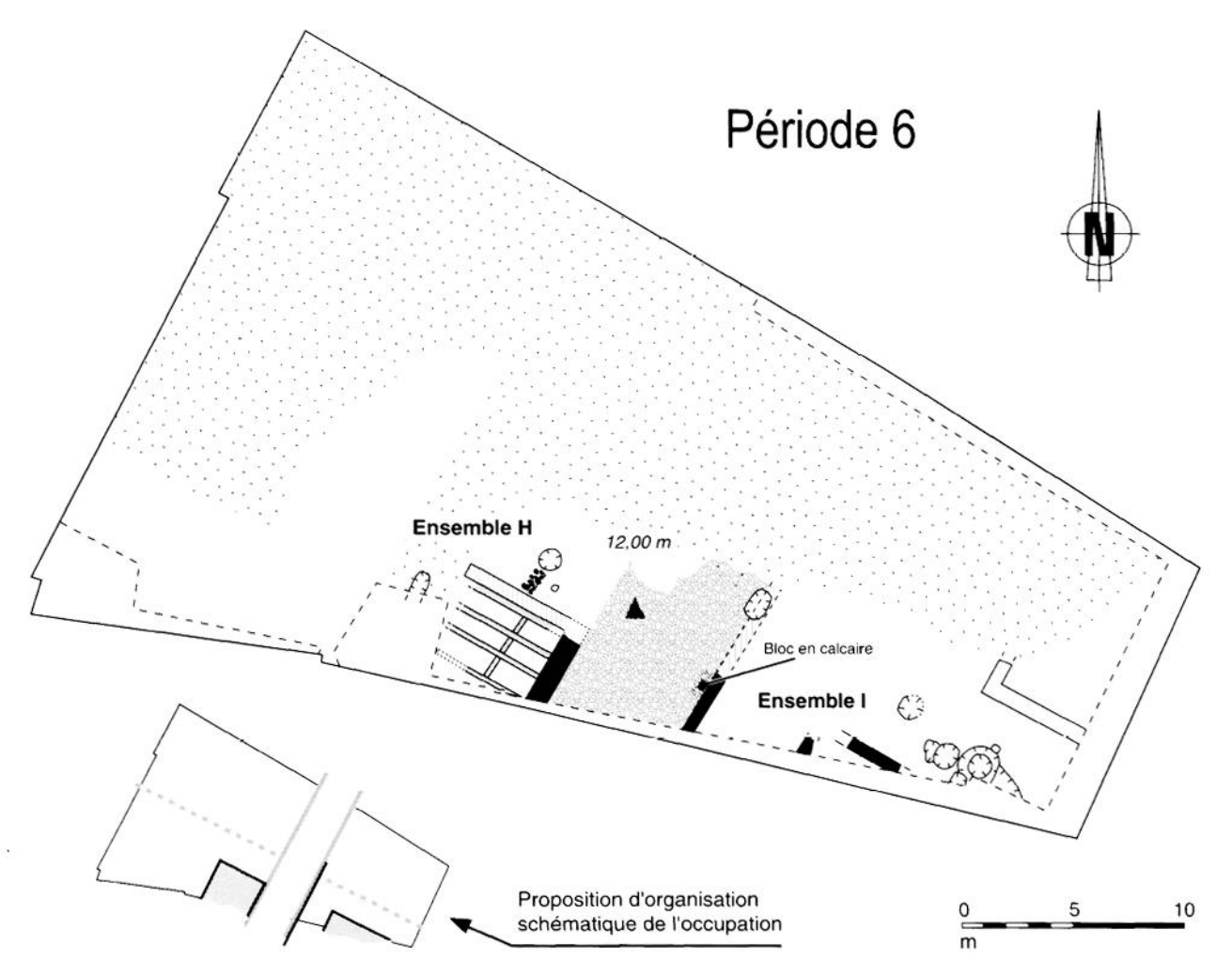

Fig. 21 : Plan des vestiges de la période 6.

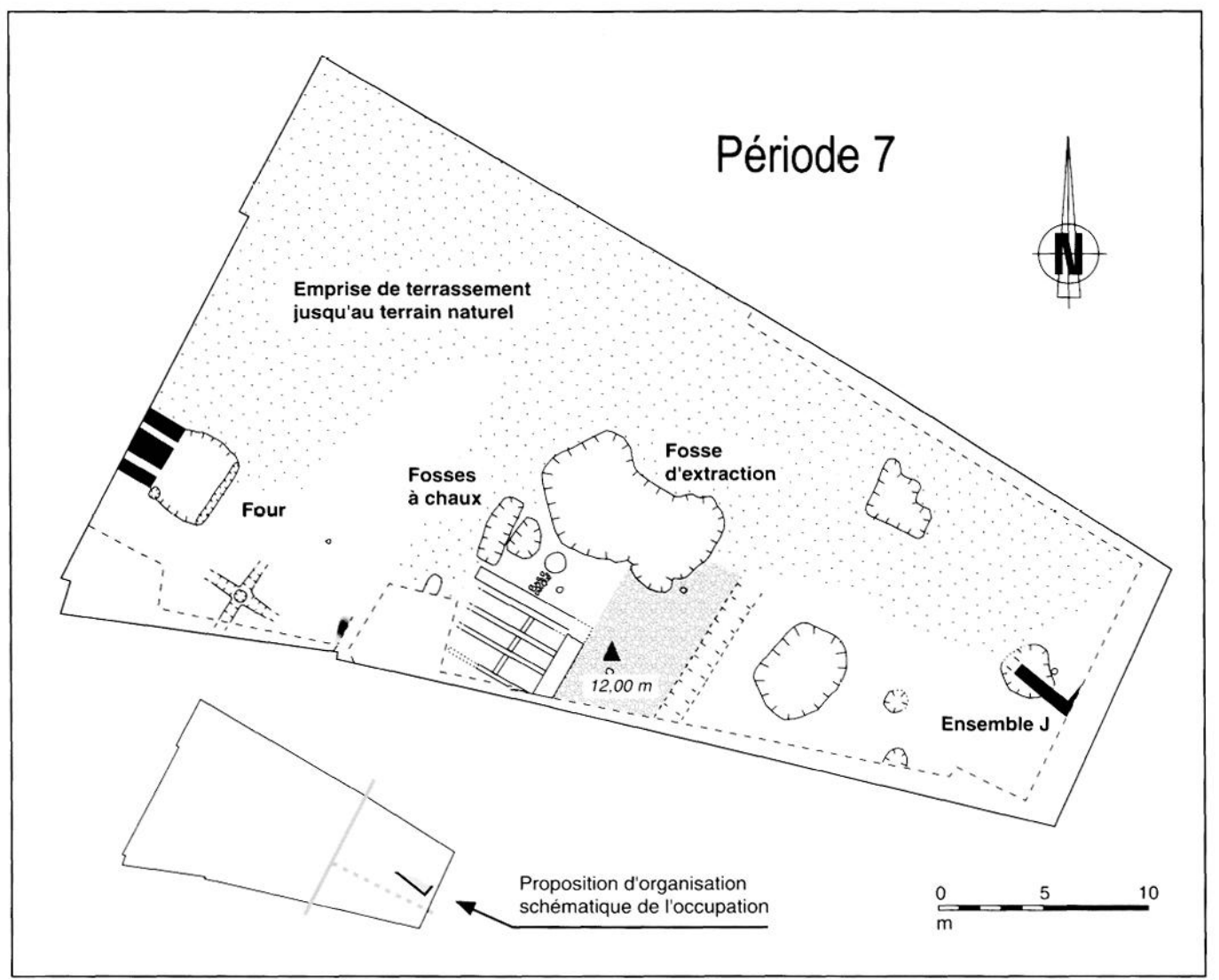

Fig. 22 : Plan des vestiges de la période 7. 


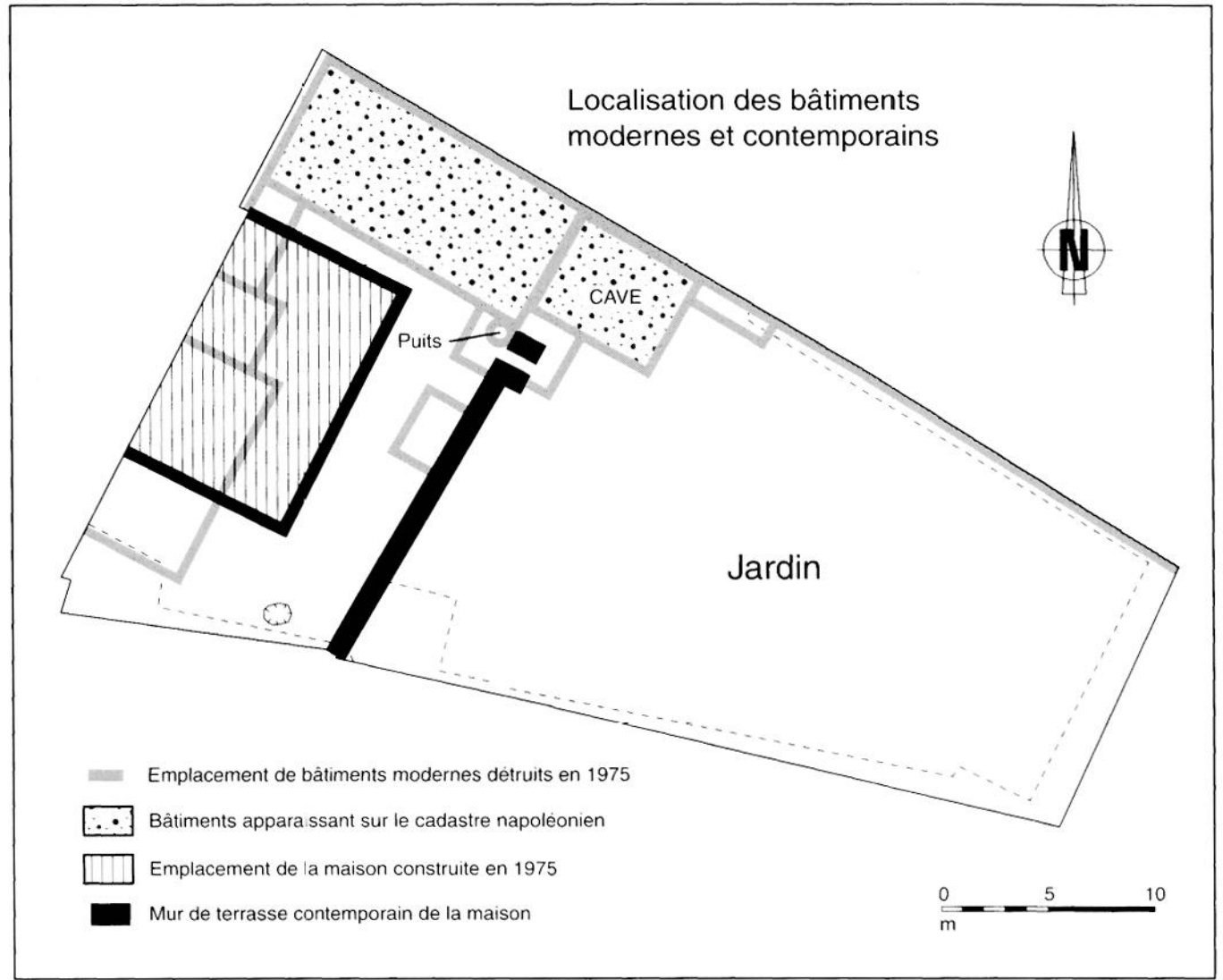

Fig. 23 : Occupations moderne et contemporaine.

ments met déjà en avant l'absence de témoignages matériels des IIIe et IVe siècles de notre ère.

\section{LE SITE APRÈS L'OCCUPATION GÁLLO-ROMAINE (Pé- riode 7)}

\section{L'oubli de l'urbanisme antique}

Plusieurs structures ne respectent plus vraiment l'organisation définie à l'époque gallo-romaine. Dans certains cas, d'importants creusements perturbent même complètement les vestiges de cette période, révélant l'abandon d'aménagements parfois essentiels comme la voirie.

\section{L'épanouissement d'une zone d'activités entre la fin du Moyen Age et l'époque moderne (fig. 22)}

La partie haute du terrain est perturbée par des terrassements liés à l'installation d'un four. Ce dernier est construit dans une grande fosse dont la partie est servait d'espace de travail. La structure clle-même se compose d'une maçonnerie en pierres de granit liées avec un mortier très sableux de couleur jaune. Les deux canaux qui y sont aménagés se poursuivent au-delà de l'emprise fouillée. L'ensemble est arasé au niveau du sol contemporain. De petits blocs de calcaire retrouvés tout autour permettraient d'identifier ici un four à chaux. Mais sa conception particulière ne correspond pas aux modèles de ce type généralement cylindriques (Flambard Héricher, 1994). Deux fosses oblongues retrouvées un peu plus à l'est viennent pourtant conforter cette hypothèse car leurs parois étaient encore tapissées de chaux. Structures de stockage ou de préparation, elles ont été complètement vidées de ce produit avant d'être comblćes par un remblai hétérogène. Le remplis- sage de l'une d'entre elles a livré un Gros « à la fleur de Lys " de Philippe VI daté de 1341-1342. Une étude plus approfondie de cet ensemble est envisagée.

Les dernières recharges de la voirie contiennent plusieurs tessons datables des XIVe-XVe siècles. Mais si l'utilisation du niveau de circulation jusqu'à la fin du Moyen Age est donc évidente, sa fonction a nécessairement changé au cours de cette période. Dans un premier temps, une réduction de la largeur de l'espace de circulation apparaît à travers un épierrement de son bord est vraisemblablement destiné à la mise en place d'un caniveau. Il est d'ailleurs possible que cet aménagement ait été réalisé dès la période 6 . Par contre, c'est bien au cours de l'époque médiévale qu'un important creusement, retrouvé au centre du terrain, recoupe l'ancienne chaussée interdisant dès lors le passage de véhicules. Quelques trous de poteaux révèlent d'ailleurs la mise en place d'installations légères sur les vestiges du niveau de circulation empierré. Ces observations indiquent que la rue gallo-romaine a été abandonnée pour être utilisée comme cour ou comme sol d'appentis à cet endroit précis.

A cette époque, le renouveau architectural qui accompagne l'épanouissement économique sous la dynastie des Montfort provoque l'ouverture de nombreuses carrières dans des terrains appartenant parfois à de simples particuliers (Leguay, 1988, 44). L'important creusement qui marque le centre du terrain fait peut-être partie de ces zones d'extractions qui seraient apparues dans beaucoup de parcelles. Deux fosses moins profondes ont été retrouvées un peu plus à l'est. Elles nc traversent pas la roche du substrat et ne peuvent ainsi correspondre à de l'extraction de granit. Leur fonction demeure indéterminée. Les rares tessons retrouvés dans le comblement de toutes ces structures confirment leur rattachement à la période médiévale. 
Plusieurs fragments de céramique " onctueuse » ont ainsi été mis au jour avec des tessons de céramique de Saintonge que l'on rencontre entre le XIIIe et le XIVe siècle ap. J.-C. Un terrassement très important a enfin été repéré sur toute la moitié nord du site. La raison de ces travaux, qui ont détruit tous les vestiges jusqu'au terrain naturel, reste également très obscure.

Une dernière construction a été mise en place dans la partie basse du terrain (ensemble J). Le seul mur conservé recoupe une fosse ayant livré des ardoises de toitures, utilisées à partir du Moyen Age dans la région. L'orientation du bâtiment est différente de celle des constructions antiques mais son emprise semble toujours respecter les limites gallo-romaines définies en période 2 . Cette observation s'explique peut-être par la pérennité d'un axe antique éventuellement situé à l'emplacement de la rue de la Tannerie. Dans ce cas, il est logique que les constructions établies le long de cette rue présentent une emprise toujours comparable à celles des bâtiments gallo-romains de tailles similaires.

\section{La reprise de l'urbanisme au XIXe siècle (fig. 23)}

Le cadastre de 1807 montre l'existence de jardins dans le terrain concerné par notre intervention. Sur ce point, la fouille a permis de comprendre qu'un apport de terre végétale a scellé les vestiges de l'occupation précédente déterminant de cette manière l'aspect du terrain tel qu'il existait encore avant notre intervention. Le nivellement de ce remblai explique son épaisseur croissante vers la partie basse. Le mur séparant le terrain de la rue de la Tannerie a certainement été construit au même moment pour soutenir la masse de terre. Sur l'ancien cadastre, deux bâtiments sont également signalés dans l'angle nord-ouest. Ils constituent le noyau originel d'un ensemble qui va se développer jusqu'à la seconde guerre mondiale. Sa nature illustre parfaitement l'image du quartier durant cette période puisqu'il s'agirait d'une maison close selon nos plus anciens visiteurs vannetais... On se souvient alors que la rue de la Tannerie abritait effectivement les deux plus importantes maisons-closes de Vannes, occupant chacune une quinzaine de filles (André, 1993, 264). L'établissement du numéro 10 va progressivement occuper toute la partie haute du terrain avant d'être démoli en 1975 pour permettre la construction d'une maison particulière. Un mur de terrasse sépare celle-ci d'un jardin conservé dans toute la partie est. Cette dernière construction a été détruite juste avant notre intervention.

\section{CONCLUSION}

La mise en place de l'urbanisme est un enjeu essentiel ici. Ses principaux caractères ne sont pourtant pas établis dès la première occupation du secteur. La fouille a clairement mis en évidence des installations initiales ne respectant aucune trame, préalable généralement nécessaire à tout développement d'une ville de tradition romaine. La mise en place d'une organisation orthogonale apparaît dans un second temps divisant alors la zone fouillée en deux îlots séparés par un espace de circulation. Ce schéma s'affirme progressivement à travers l'évolution du bâti ou la construction d'une chaussée. Vraisemblablement défini sous le règne d'Auguste, il demeure le même durant toute l'Antiquité.
Plusieurs indices permettent également une approche de la place du quartier au sein de l'agglomération vénète. En effet, si les aménagements successifs de l'îlot est n'offrent guère d'informations sur la nature de l'occupation, à l'inverse l'établissement d'un ensemble public doit être envisagé dans l'îlot ouest vers la fin du Ier siècle de notre ère au plus tôt. Une telle suggestion renforcerait un lien possible avec le complexe architectural retrouvé directemont au nord. Cette idée s'appuie également sur l'appartenance de toutes ces constructions imposantes (de même facture) à une organisation commune. Toutes ces hypothèses restent cependant fragiles car les vestiges conservés n'offrent pas d'information réellement décisive. Des axes de recherches sont néanmoins proposés. Les données recueillies au 10, rue de la Tannerie sur la mise en place et l'évolution de l'urbanisme offrent une base de départ qu'il faudra confronter aux autres découvertes. La place, l'organisation et le rôle d'un projet monumental très vaste au coeur d'une cité apparemment limitée doivent également être cernés. Enfin, l'occupation qui suit la destruction des ensembles du Haut-Empire mérite une attention particulière dans ce contexte typiquement vannetais d'un déplacement du noyau urbain sur la colline voisine.

\section{REMERCIEMENTS}

Remerciements à Laure Simon pour l'identification des céramiques antiques, à Laurent Beuchet pour celle des céramiques médiévales et modemes et à Dominique Pouille pour celle des monnaies.

\section{BIBLIOGRAPHIE}

ANDRE, B., 1993 - Vannes, ville de garnison de 1872 à 1914 : Mariage d'amour ou de raison. In 2000 ans d'histoire de Vannes. Archives municipales, Vannes.

BEDON, R., CHEVAlLIER, R., et PINON, P., 1988 - Architecture et urbanisme en Gaule romaine. Paris.

BEDOYERE, G. de la, 1992 - Roman Towns in Britain. Londres.

CATALOGUE VANNES 1992 - Quand Vannes s'appelait Darioritum. La Cohue, Musée de Vannes. Juin 1992 - Décembre 1993.

CRUMMY, N., 1983 - Colchester archaeological report 2 : The Roman small finds from excavations in Colchester 1971-9. Colchester.

DESBORDES, J.-M., et LOUSTAUD, J.-P., 1992 - Limoges. $2 e$ colloque AQUITANIA. Villes et agglomérations urbaines antiques du sud-ouest de la Gaule. Histoire et archéologie. Bordeaux, 1992, 110-121.

DE CUSSE, L., 1877 - Notes sur une nécropole gallo-romaine découverte à Vannes en 1876. Bulletin de la Société Polymathique du Morbihan, 98-101.

DERU, X., et FELLER, M., 1996 - Horizons chronologiques de la céramique du Haut-Empire dans le quartier du Pontiffroy à Metz (Moselle). In Actes du Congrès de Dijon. SFECAG, 433-459.

DESBAT, A., 1985 - La région de Lyon et de Vienne. In Lasfargues J. et al., Architecture de terre et de bois, l'habitat privé des provinces occidentales du monde romain. Antécédents et prolongements : Protohistoire, Moyen Age et quelques expériences contemporaines. $D A F, 2,75-83$.

FAUDUET, I., 1993 - Les temples de tradition celtique en Gaule romaine. Paris.

FEUGERE, M., 1985 - Les fibules en Gaule méridionale de la conquête à la fin du Ve siècle ap. J.-C.. Rev. Archéo. de Narbonnaise, supplément $n^{\circ} 12$ 
FEVRIER, P.-A., 1992 - Approches récentes du fait urbain dans les Gaules. $2 e$ colloque AQUITANIA. Villes et agglomérations urbaines antiques du sud-ouest de la Gaule. Histoire et archéologie. Bordeaux, 1992, 177-190.

FLAMBARD HERICHER, A.M., 1994 - Un four à chaux de la fin du Moyen Age au Molay-Littry (Calvados). Archéologie Médiévale, XXIV, 367-377.

GIRARDY-CAILLAT, C., 1992 - Périgueux (Dordogne), Vesunna, Civitas Patrocoriorum. $2 e$ colloque AQUITANIA. Villes et agglomérations urbaines antiques du sud-ouest de la Gaule. IIistoire et archéologie. Bordeaux, 1992, 125-129.

GOUDINEAU, C., 1968 - La ceramique arétine lisse. Fouilles de l'école française de Rome à Bolsena (Poggio Moscini) 19621967. Paris.

LEGUAY, J.P. et aL, 1988 - Histoire de Vannes et de sa région. Toulouse.

MARSILLE, H., 1982 - Les Maures vénètes. Bulletin de la société polymathique du Morbihan, 109, 13-24.
MENEZ, Y., 1985 - Les céramiques fumigées de l'ouest de la Gaule. Cahiers de Quimper antique, 2.

MENEZ, Y., DAIRE, M.-Y., HYVERT, J., LANGOUET, L., LE BIHAN, J.-P. et TANGUY, D., 1990 - Les bâtiments de l'Age du Fer en Armorique. In Duval, A. \& Le Bihan, J.-P. \& Menez, Y., Les Gaulois d'Armorique, la fin de l'Age du Fer en Europe tempérée, Actes du XIle colloque AFEAF, Rev. Archéo Ouest, supplément $n^{\circ} 3,121-137$.

PAUNIER, D., 1985 - La Suisse. In Lasfargues J. et al., Architecture de terre et de bois, l'habitat privé des provinces occidentales du monde romain. Antécédents et prolongements : Protohistoire, Moyen Age et quelques expériences contemporaines. $D A F, \mathbf{2}$, 113-126.

WACHER, J.S., 1985 - La Bretagne : généralités. In Lasfargues J. et al., Architecture de terre et de bois, l'habitat privé des provinces occidentales du monde romain. Antécédents et prolongements : Protohistoire, Moyen Age et quelques expériences contemporaines. $D A F, 2,143-151$.

WIGHTMAN, E. M., 1970 - Roman Trier and the Treveri. Londres. 